\title{
Analysis on conservation of disulphide bonds and their structural features in homologous protein domain families Ratna R Thangudu ${ }^{1,4}$, Malini Manoharan ${ }^{2}$, N Srinivasan ${ }^{3}$, Frédéric Cadet ${ }^{1}$, R Sowdhamini*2 and Bernard Offmann*1
}

\begin{abstract}
Address: ${ }^{1}$ Laboratoire de Biochimie et Génétique Moléculaire, Université de La Réunion, BP 7151, 15 avenue René Cassin, 97715 Saint Denis Messag Cedex 09, La Réunion, France, ${ }^{2}$ National Centre for Biological Sciences, GKVK Campus, Bangalore, India, ${ }^{3}$ Molecular Biophysics Unit, Indian Institute of Science, Bangalore 560 012, India and ${ }^{4}$ National Center for Biotechnology Information, U.S. National Library of Medicine, 8600 Rockville Pike, Bethesda, MD 20894, USA

Email: Ratna RThangudu - thangudr@ncbi.nlm.nih.gov; Malini Manoharan - malinim@ncbs.res.in; N Srinivasan - ns@mbu.iisc.ernet.in; Frédéric Cadet - frederic.cadet@univ-reunion.fr; R Sowdhamini* - mini@ncbs.res.in; Bernard Offmann* - bernard.offmann@univ-reunion.fr

* Corresponding authors
\end{abstract}

Published: 26 December 2008

BMC Structural Biology 2008, 8:55

doi:10.1 I 86/I472-6807-8-55
Received: 4 May 2008

Accepted: 26 December 2008

(c) 2008 Thangudu et al; licensee BioMed Central Ltd.

This is an Open Access article distributed under the terms of the Creative Commons Attribution License (http://creativecommons.org/licenses/by/2.0), which permits unrestricted use, distribution, and reproduction in any medium, provided the original work is properly cited.

\begin{abstract}
Background: Disulphide bridges are well known to play key roles in stability, folding and functions of proteins. Introduction or deletion of disulphides by site-directed mutagenesis have produced varying effects on stability and folding depending upon the protein and location of disulphide in the 3-D structure. Given the lack of complete understanding it is worthwhile to learn from an analysis of extent of conservation of disulphides in homologous proteins. We have also addressed the question of what structural interactions replaces a disulphide in a homologue in another homologue.
\end{abstract}

Results: Using a dataset involving 34,752 pairwise comparisons of homologous protein domains corresponding to 300 protein domain families of known 3-D structures, we provide a comprehensive analysis of extent of conservation of disulphide bridges and their structural features. We report that only $54 \%$ of all the disulphide bonds compared between the homologous pairs are conserved, even if, a small fraction of the non-conserved disulphides do include cytoplasmic proteins. Also, only about one fourth of the distinct disulphides are conserved in all the members in protein families. We note that while conservation of disulphide is common in many families, disulphide bond mutations are quite prevalent. Interestingly, we note that there is no clear relationship between sequence identity between two homologous proteins and disulphide bond conservation. Our analysis on structural features at the sites where cysteines forming disulphide in one homologue are replaced by non-Cys residues show that the elimination of a disulphide in a homologue need not always result in stabilizing interactions between equivalent residues.

Conclusion: We observe that in the homologous proteins, disulphide bonds are conserved only to a modest extent. Very interestingly, we note that extent of conservation of disulphide in homologous proteins is unrelated to the overall sequence identity between homologues. The nonconserved disulphides are often associated with variable structural features that were recruited to be associated with differentiation or specialisation of protein function. 


\section{Background}

Cysteine residues assume important role in proteins through a wide range of functions such as disulphide bond formation, metal binding, electron donation, hydrolysis and redox catalysis. Disulphide bond formation is one of the most important post-translational modification events of a protein in the biological cell. Disulphide bond stabilization of a protein is considered to be entropy driven through destabilization of the unfolded state and may also contribute enthalpically through favourable local interactions like compacting the clusters of hydrophobic residues. Knowledge of disulphide bond connectivity is influential in protein folding experiments and in 3-D structure prediction.

Since Richardson's and Thornton's extensive and detailed analysis on disulphide bonds in 1981, several studies have been reported on the oxidation state of cysteines and the conservation, connectivity and structure of disulphide bonds $[1,2]$. Several computational methods have been developed to predict or model cysteine sidechains that might be involved in disulphide formation [3-17] and also to identify their connectivity patterns in multiple disulphide bond containing proteins [18-24]. Tools are available to model disulphide bonds in proteins by estimating the local stereochemical compatibility to accommodate a disulphide bond $[25,26]$.

Disulphide bonds are generally believed to be conserved among related proteins $[1,27]$ and the cystine connectivity pattern may be used as a diagnostic to identify proteins of similar 3-D structure. An inverse approach starting with clearly related proteins aims to identify cystine connectivity pattern using sequence alignments[28]. Mas and coworkers have derived relationship amongst even nonhomologous proteins belonging to different superfamilies [29]. They explained the antagonistic properties of potato carboxypeptidase inhibitor against growth factors by comparing its structural features with epidermal growth factor, derived through their disulphide bridge topology even when their connectivity differs [29]. The conservation of disulphide bond connectivity patterns, enable the identification of remote homologues even when the most popular sequence search methods may fail to do so. Such approaches are complicated by observations of topologically equivalent disulphide bonds in non-homologues and also by non-equivalent number of disulphide bonds in close homologues [29].

Many studies examined the role of disulphide bonds in the protein structure and function, some through mutagenesis experiments [30-38], while a few others studied the same in evolutionary perspective [39]. Thornton observed that in protein superfamilies the conservation rules appear less stringent [2] from the analysis of limited data. Non-conservation in such cases is usually associated with loss of both the cysteines involved in the disulphide bond $[1,2]$. Amino acids linked by a specific role mutate in a coordinated manner if the geometry of the contacts are the same in all the proteins [40]. Kreisberg and coworkers employed multiple sequence alignments to map patterns of correlated cysteine mutations to identify positions of protein disulphide bonds [41].

The high levels of structural and functional similarity among several distantly homologous proteins overwhelm protein classification methods that rely on simple sequence identity. The SCOP [42] database contains several families with mean pairwise sequence identity among the members of the family well below $40 \%$. Hence, there could be significant changes in structure or function due to the presence or absence of one or more disulphide bonds in a protein compared to its homologue. This necessitates a comprehensive study of disulphide bond variations in homologous protein domains.

It has been documented that ligand bound cysteines are more similar to disulphide bonded cysteine than free cysteines $[7,8]$. However, we have confined the present study to disulphide bonded cysteines as many of the disulphide bond containing proteins are extracellular or secreted whereas there are many intracellular proteins with cysteines liganded to metal ions (such as zinc coordinated cysteines in transcription factors which are nuclear proteins),

A systematic study of structural conservation of disulphide bonds in homologous protein domains should reduce the limitations of sequence comparison in principle. But this raises several interesting questions: to what extent sequence similarity guarantees the conservation of disulphide bonds in related proteins? What is the structural or functional consequence of non-conservation? Do the locations of non-conserved disulphide bonds exhibit any similarities? To address these questions, we attempted a large-scale analysis of conservation of disulphide bonds in the homologous families of SCOP [42]. In principle, it will be more definitive as well as interesting to uplift the entire analysis after including sequence homologues. However, it was imperative that we restrict the current analysis to structural entries within homologous families only so that we do not have to compensate for the quality of alignments nor make any assumptions on disulphide bond connectivity in the homologues of yet unknown structure. Understanding the consequences of such natural variations in native proteins should help in overcoming the unfavourable effects of mutational experiments, like disruption of native structure $[43,44]$. Implications of this analysis for prediction of disulphide connectivity and protein modelling are also discussed. 


\section{Results \\ Distribution of disulphide bonds in homologous protein families}

Our dataset for analysis comprises a total of 300 homologous families from SCOP, each populated with at least two members and where at least one of them is annotated with a disulphide bond. A total of 3990 disulphide bonds are present in these 300 families. The distribution of disulphide bonds in different fold classes is depicted in Table 1. Disulphide bonds are common in all fold classes, although in varying degrees. However, only $22 \%$ of the families in SCOP contain annotated disulphide bonds. A closer look at these families might provide clues about their preferential distribution. Small proteins with less than 70 residues, by the SCOP definition, are usually rich in disulphide bonds which are known to contribute critically to their stability[45] since small proteins usually lack a strong hydrophobic core[46]. Disulphides are known to exist in most small proteins $[47,48]$. Indeed, a large majority $(67 \%)$ of the small protein families in SCOP 1.67 have disulphide bonds (Table 1).

On the other hand, all- $\alpha$ proteins showed least preference for these crosslinks, probably because of the steric hindrances involved in the formation of disulphides between cysteines present in different helices or same helix. The repertoire of cysteine conformations suggests preferences in disulphide distribution within beta sheets [49]. The distribution of disulphide bridges in terms of secondary structure highlights their preference for stabilizing strands and loops (see later).

\section{Analyzing disulphide conservation using relaxed criterion} In our study, a disulphide bond is considered strictly conserved in a multiple alignment if both the cysteines involved are placed at equivalent positions in all the sequences in the alignment. However, due to slight changes in the conformations, rigid body shifts and uncertainties in the structure-based alignments some times potentially equivalent cystines from homologues may not align perfectly. Hence, according to the relaxed criteria, an arbitrary shift of 4 residues on either side of the cystine positions is used for counting the distinct disulphide bonds.

A total of 34752 pairwise comparisons between members within each 300 families were analysed. The number of conserved disulphide bonds in these homologous pairs rose from 19500 to 26065 when the relaxation was applied. The increase, although marginal, indeed points out to the potential structural variation in homologous proteins. Only $54 \%$ of all the disulphide bonds compared between the homologous pairs are conserved.

\section{A small fraction of the proteins with cytoplasmic localisation lack conserved disulphide bonds}

Only 52 proteins in the current dataset, predicted to be cytoplasmic (see Additional files), were reported to be cytoplasmic after extensive literature searches. This amounts to a minor fraction (2\%) of proteins in the entire dataset employed for the analysis. More importantly, only 38 proteins in the current dataset are reported to be cytoplasmic and do not share conservation of disulphide bonds $(1.5 \%)$, suggesting that the dataset is not likely to be biased by protein families with diverse cellular localisation that can affect the low conservation of disulphide bonds. Most of such rare and peculiar examples are oxidoreductases like thioredoxins where disulphides are essential for their function.

In the family based multiple structural alignments, all the distinct disulphide bonds in a given family were identified

Table I: Fold distribution of disulphide bond containing families under study in SCOP and number of disulphides and distinct disulphides the database features.

\begin{tabular}{|c|c|c|c|c|c|c|c|}
\hline \multirow[b]{2}{*}{ SCOPa Class } & \multicolumn{2}{|c|}{$\begin{array}{l}\text { Number of disulphide bond } \\
\text { containing families }\end{array}$} & \multicolumn{3}{|c|}{$\begin{array}{l}\text { Number of members per family in } \\
\text { ANALYCYS }\end{array}$} & \multicolumn{2}{|c|}{ Number of disulphides } \\
\hline & SCOP & ANALYCYS & 2 & $3-10$ & $>10$ & $\begin{array}{l}\text { No of } \\
\text { disulphides }\end{array}$ & $\begin{array}{l}\text { No of distinct } \\
\text { disulphides }\end{array}$ \\
\hline All $\alpha(550)^{c}$ & 65 & 38 & 12 & 17 & 9 & 524 & 125 \\
\hline All $\beta$ (529) & 167 & 89 & 19 & 46 & 24 & 961 & 256 \\
\hline$\alpha / \beta$ (593) & 111 & 58 & 8 & 30 & 20 & 427 & 192 \\
\hline$\alpha+\beta(650)$ & 106 & 59 & 16 & 30 & 13 & 640 & $|4|$ \\
\hline $\begin{array}{l}\text { Small proteins } \\
(162)\end{array}$ & 108 & 56 & 9 & 35 & 12 & 1438 & 262 \\
\hline Total $(2630)$ & 580 & 300 & 64 & 158 & 78 & 3990 & 976 \\
\hline
\end{tabular}

a Structural classification of proteins.

b Database generated in the current analysis of disulphide conservation in SCOP families.

$C$ The numbers between parentheses represents the total number of families in the different SCOP class 
and their conservation state were estimated (see Materials and Methods). Overall, a total of 976 distinct disulphides were featured across all 300 families in our dataset. The total number of distinct disulphides per family varies from 1 to 15 with an average of 3.33 disulphides (Figure. 1 ). About one third of the families (108) possess only a single distinct disulphide. The number of distinct disulphides in small proteins ranges from 1 to 15 but with an average of 4.53. A total of 14 families display more than 10 distinct disulphides. The highest number of distinct disulphides that was found in a family is 15 and was found in the family of sialidases/neuraminidases (SCOP code: b.68.1.1), the beta-glycanases family (SCOP family code: c.1.8.3), growth factor receptor domain (SCOP family code: g.3.9.1) and the transferrin family (SCOP family code: c.94.1.2). Since this classification is at individual domain level, we might not encounter highly disulphiderich multi-domain systems, such as wheat-germ agglutinin or serum albumin-like folds.

We further classified the distinct disulphides for their conservation into three categories: high $(\mathrm{H})$, medium $(\mathrm{M})$ and low (L) conserved disulphide bonds. As shown in Fig- ure. 2, their distribution in different fold classes, clearly establishes interesting facts. While the majority of the disulphide bonds in small proteins are highly conserved, re-establishing the fact that disulphide bonds play a strong role in their structural stabilization, they are less conserved in other fold classes. Hence, in general, conservation of disulphide bonds among homologues of globular proteins is only modest.

Further, analysis of the half-cystine containing regions was performed to investigate whether the associated local structures were topologically conserved (see Material and Methods) in homologous proteins along with the disulphide bond itself. Our analysis suggests that in about 70\% of the disulphide bonds, at least one of the half-cystine is localized in structurally conserved region. This is rather interesting since disulphide bonds are reputed to occur preferentially in loop regions or connect regular secondary structures with coiled regions[7] (see later). The highly variable nature of loops/coils did not deter the conservation of local structures where the disulphides are found, strongly suggesting their role in structural stabilization. Similarly, in almost $80 \%$ of the disulphide bonds where

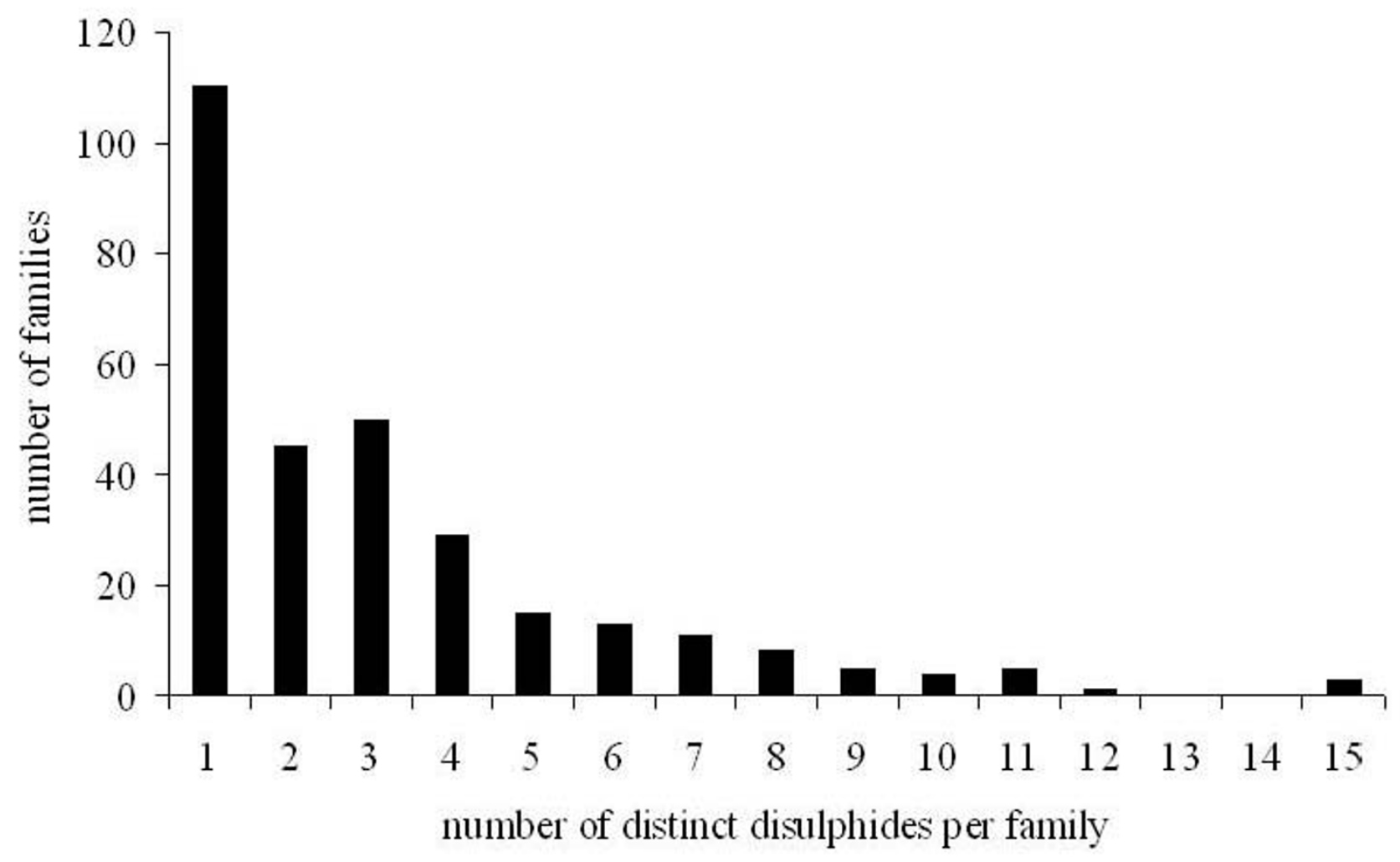

Figure I

Distribution of total number of distinct disulphides per family. 


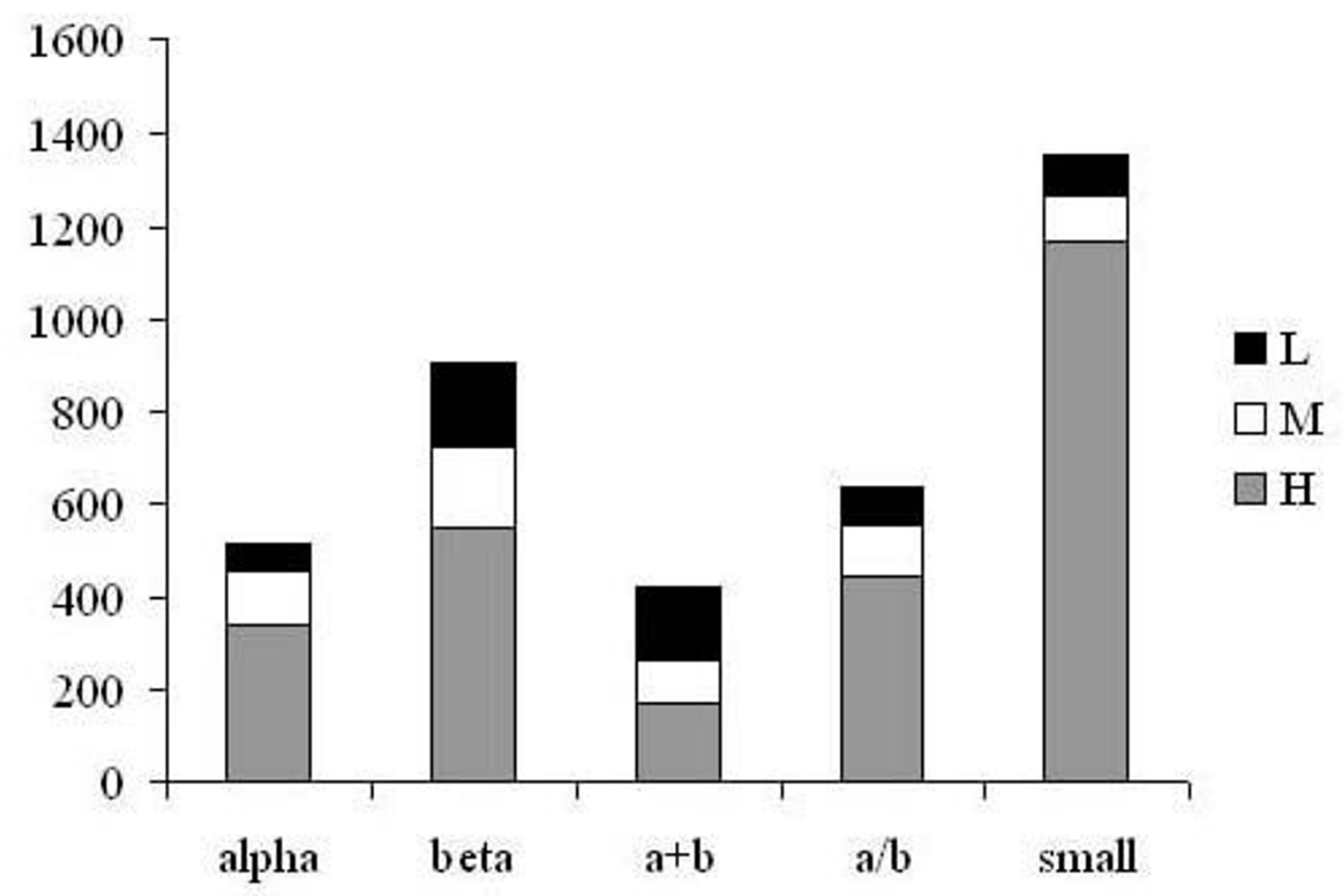

Figure 2

Distribution of highly (H), medium (M) and low (L) conserved disulphides in SCOP database.

the local structures of the participating half-cystines are in structurally variable regions, at least one half-cystine is in coil state.

Low-conserved disulphide bonds stabilise insertion regions specific to a subfamily

When the conservation status of the disulphides was analysed, we found that most of the highly conserved disulphide bonds have at least one half-cystine in topologically equivalent region. However, in poorly conserved disulphide bonds, more than $50 \%$ of their half-cystines lie in structurally variable regions. This suggests that a low-conserved disulphide bond in a family member is an adaptation and usually tends to constrain highly structurally variable regions of the family. For example, trypsin-like serine proteinases share a common serine proteinase fold, and often have a sequence identity below 30\% with diverse functions. The poorly conserved disulphide bonds in this family appeared to stabilise insertion regions specific to a subfamily. The 12-218 (alignment positions) disulphide bond in Factor $\mathrm{B}(\mathrm{C} 1-\mathrm{C} 122)$ associates the insertion, $\mathrm{N}$-terminal linker to the main body of the serine proteinase [50] and is highly conserved only in plasminogen and chymotrypsinogen[51] The helix-turn-helix insertion (T125a-D133) unique to factor B SP (1dle) is stabilised by constraining through the distinct 221-241 disulphide bond (C125-C125p)[50]. The 156-489 bridge is specific to snake venom serine proteinases, clamps the C-terminal tail to the 99-loop that surrounds the active site[52]. All these three poorly conserved distinct disulphide bonds of this family did not have any topologically equivalent alignment partners in non-conserved members and had at least one of the cysteine aligned with a gap. Another spectacular example is the melanoma inhibitory activity (MIA) protein. It is the first structure of a secreted protein with a Src homology 3 (SH3)-like domain with $\mathrm{N}$ and C-terminal extensions of about 20 residues each adding additional structural elements, forming a previously undescribed fold. The 12-17 and 35-106 disulphide bonds, unique to this protein scaffold, stabilise loop regions and are not observed in classical SH3 domains. The structure also suggests a likely protein interaction site and suggests that, unlike conventional SH3 domains, MIA does not recognize polyproline helices and may have evolved with different functions[53].

\section{Disulphide conservation versus number of disulphide bonds per family}

Totally conserved disulphides are expected to play an important role in the structure and/or function of homol- 
ogous proteins. Also, it is of interest to analyze the structural and functional roles of single disulphides that are featured in some of the protein families. Here, we assess the possible relationship between extent of disulphide conservation and 'disulphide richness' in protein families.

As shown in the previous section, not all the disulphide bonds in a family are equally conserved. One fourth of the all the distinct disulphides (237 out of 948) are totally conserved (i.e. in all the family members). Interestingly, the topological state of a vast majority of these 237 fully conserved disulphides is also highly conserved. The topological equivalence of cysteine residues participating in disulphide bond in family of proteins inherently highlights the conservation of structural state (secondary structure) and also solvent accessibility. These fully conserved disulphides, as shown in Figure. 3, are distributed in as much as 33\% (100 families) of the total number of families in our dataset thus indicating that full conservation of disulphides is not widespread but is not rare either. The maximum number of fully conserved disulphides that a family displays in our dataset was eight (Figure. 3) and was featured in osmotin thomatin-like protein family (b.25.1.1) and in glycosyl hydrolase family 7 catalytic core (b.29.1.10). In the former, all eight distinct disulphides are indeed fully conserved and the pairwise sequence similarities between all four individual members varied between $54.7 \%$ and $79.5 \%$. In the latter, 8 out of 10 distinct disulphides that are featured in the family are fully conserved and the pairwise sequence similarity between all four members in the family ranged from $40.5 \%$ and $56.8 \%$.

Further analysis of the content of fully conserved disulphides out of the total number of distinct disulphides per family, as illustrated in Figure. 3, shows that a total of 51 families are found to have all their disulphides conserved (diagonal on the plot). However, out of these 51 families, $50 \%$ have only 2 members in the data set and almost $90 \%$ of them have $\leq 4$ members. It is noteworthy that the other 49 families also contain other disulphide bonds with variable conservation. The fraction of single disulphide bonded families where the disulphide is fully conserved is very less, i.e. 15 out of 111 families (Table 2).

\section{Conservation status of disulphide bonds versus sequence identity in pairwise comparisons}

During the course of evolution, considerable changes in the amino acid sequences may occur in homologous proteins usually retaining the function. When not functionally relevant, disulphide bonds act as structural scaffolds in small proteins, impose geometrical restraints and reduce entropy during folding, or provide additional stability to flexible regions liable under extreme conditions of $\mathrm{pH}$ or temperature. Hence, one might argue that disulphide bonds are strongly conserved in homologous pro- teins sharing higher sequence similarity. To investigate this relationship, we have plotted pairwise sequence identity against pairwise disulphide bond conservation (Figure. 4).

As demonstrated in the previous sections, non-conservation of disulphides is a common feature though, given the importance of disulphide bonds, one would have expected their good conservation throughout the range of sequence identities. But, as shown in Figure. 4, the spread of the data points in the different disulphide conservation ranges namely beyond the $50 \%$ or even above the $75 \%$ sequence similarity mark suggests the opposite. This is interesting since the structurally and functionally important features (like disulphides) in closely related proteins may be expected to be well conserved. It should be noted here that care has been taken, when setting up the dataset, to remove engineered or mutant proteins and hence these protein entries with high sequence identity are natural homologues. On the other hand, the entire range of disulphide conservation is densely populated below the $40 \%$ sequence identity mark. A high fraction of disulphide conservation at lower sequence similarity points to the selective evolutionary pressure to preserve the fold, whereas non-conservation can be readily attributed to sequence divergence or recruitment of additional structural features. In either case, it clearly emerges that not all disulphide bonds are equally important even in closely related proteins. The highly conserved disulphide bonds are the ones that would play a crucial role in the folding process or function of the protein, while the rest would have protein specific roles.

Disulphide bonds are known to be present mainly in the extracellular proteins and rare in the cytoplasmic compartments of most organisms, due to the reductive nature of the cytosol [54,55]. In bacteria they are usually restricted to extra-cytoplasmic compartments with a more oxidative nature or secreted into the media, and in eukaryota the endoplasmatic reticulum or secreted into the external milieu.

Although rare, there are examples of disulfide bonds in intracellular proteins. They are found mainly in proteins that catalyze oxidation-reduction (redox) processes. For example, in the members of thoiredoxin family, thioredoxin and glutathione reductase, a disulfide bond forms during part of a catalytic cycle, and Hsp33 and OxyR, in which a disulfide bond forms as part of a redox-sensing mechanism [56-58]. These functionally important disulphide bonds are usually very highly conserved even as the family members share very low sequence identity (Figure 4 and above discussion). But such intracellular disulfide bonds are rare and generally transiently formed or marginally stable, rather than being essential for structural integrity. 


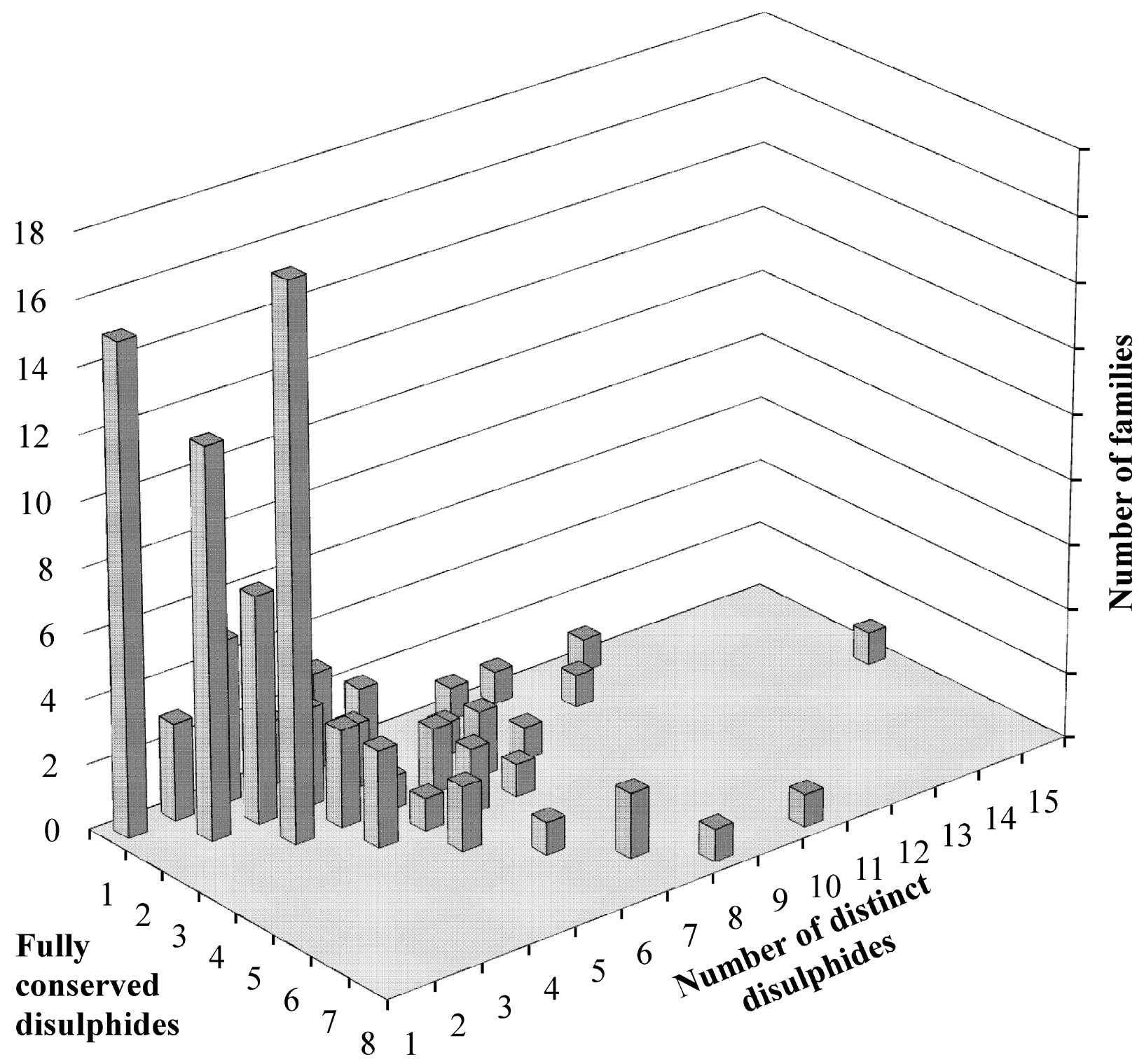

Figure 3

Analysis of fully conserved disulphides in SCOP families. Distribution of number of fully conserved disulphides against number of distinct disulphides per family.

However an exception to this rule is the unexpected discovery of three intra-chain disulfide bonds in archeal adenylosuccinate lyase (ASL) from Pyrobaculum aerophilum [59]. These disulfide bonds are poorly conserved and the absence of these disulfide bonds in ASLs from other species implies that the $P$. aerophilum enzyme acquired (or retained) its disulfide bonds during evolution to increase its stability to extreme environmental conditions. Similarly, the disulphide bond in C-terminal domain TATA-box binding protein from Archaeon Pyrococcus woesei (SCOP family code: d.129.1.1) is absent in other species.

The lack of correlation between disulphide conservation and sequence identity, however, raises another interesting question i.e. is there a relationship between the extent of conservation of disulphide bonds and disulphide richness? Richness or abundance of disulphides is calculated 
Table 2: Characteristics of families possessing a single distinct disulphide that is fully conserved across all members.

\begin{tabular}{|c|c|c|c|c|}
\hline SCOP family code & SCOP family name & $\begin{array}{l}\text { Number of proteins in } \\
\text { the family }\end{array}$ & $\begin{array}{l}\text { Range of sequence } \\
\text { similarity }\end{array}$ & Solvent accessibility \\
\hline a.137.2.1 & $\begin{array}{l}\text { Methanol dehydrogenase } \\
\text { subunit }\end{array}$ & 4 & $65.2-73.7$ & Exposed \\
\hline a.152.1.2 & Hypothetical protein TMI620 & 2 & 100 & Exposed \\
\hline a.33.l.I & Ectatomin subunits & 2 & 47.1 & Buried or partially exposed \\
\hline b.I.I8.4 & $\begin{array}{l}\text { Class II viral fusion proteins C- } \\
\text { terminal domain }\end{array}$ & 2 & 29.3 & Buried \\
\hline b. 12.1 .2 & Colipase-binding domain & 4 & $53.2-83.1$ & Exposed \\
\hline b.16.I.I & Ecotin, trypsin inhibitor & 2 & 95.4 & Exposed \\
\hline b.18.1.2 & $\begin{array}{l}\text { Discoidin domain (FA58C, } \\
\text { coagulation factor } 5 / 8 \mathrm{C}- \\
\text { terminal domain) }\end{array}$ & 3 & $38.2-42$ & Partially exposed \\
\hline b.2.2.I & $\begin{array}{l}\text { Cellulose-binding domain } \\
\text { family II }\end{array}$ & 3 & 29.5 & Buried or partially exposed \\
\hline b.2.3.5 & FI7c-type adhesin & 2 & 91.4 & Partially exposed \\
\hline b.29.1.5 & Pentraxin (pentaxin) & 2 & 51.5 & Buried \\
\hline b.6I.2.I & Metalloprotease inhibitor & 2 & 38 & Partially exposed \\
\hline b.78.I.I & $\begin{array}{l}\text { alpha-D-mannose-specific } \\
\text { plant lectins }\end{array}$ & 5 & $48.1-85.2$ & Buried \\
\hline c.69.1.24 & $\begin{array}{l}\text { Dipeptidyl peptidase IV/CD26, } \\
\text { C-terminal domain }\end{array}$ & 2 & 92.6 & Buried \\
\hline d. 165.1 .2 & Shiga toxin, A-chain & 2 & 57.3 & Partially exposed \\
\hline d.233.1.I & $\begin{array}{l}\text { Inhibitor of vertebrate } \\
\text { lysozyme, Ivy }\end{array}$ & 2 & 19.7 & Partially exposed \\
\hline
\end{tabular}

as the number of distinct disulphides compared between a pair of proteins divided by the average length of their sequences. When plotted, the dispersion of data points across the range of conservation clearly indicated there exists no correlation between conservation and the disulphide richness (data not shown). Hence, from Figure. 4, two distinct situations can be expected: (i) non-conservation of disulphide bonds in homologues with high sequence identity and (ii) high conservation of disulphides in homologues with low sequence identity. With respect to this, we have performed a thorough analysis of several families of proteins and collected literature to understand these two situations.

(i) non-conservation of disulphides in homologues with high sequence identity

In the current analysis, several cases of homologues with high sequence identity but with non-conservation of disulphide bonds have been observed. We specifically studied several such cases to understand the reasons for their absence/appearance. The reasons are presented below and in Table 3.

We have identified several naturally occurring non-conserved disulphide bonds in close homologues, which may play a significant role in their structural or functional differences. These disulphide bonds significantly contribute towards the protein structure or function (see Table 3 ) through oligomerization $[60,61]$ or increased activity [6264] or by bringing about local structural compaction[65] yet with little effect on the conformational stability of the overall fold. These disulphide bonds provide local structural stabilization usually in conjunction with other features like clustering of hydrophobic residues.

It is, however, not always immediately possible to ascertain role of such disulphides in related proteins [66-69]. An additional disulphide bond may not have direct effect on the functional difference between the homologues, although it brings about local structural variation.

(ii) high conservation with low sequence identity

Thioltransferases (c.47.1.1) are the members of thioredoxin superfamily, with a highly conserved fold and two vicinal (CXXC) active site cysteine residues and mediate formation of disulphides (oxidized form) or dithiols (reduced form). This class of enzymes mediates oxidation and reduction of disulphide bonds through thioldisulfide exchange reactions between the active site cysteines of these enzymes and the cysteines in the target protein. Some of the members of this family, for example, thiredoxin from Anabaena sp. (PDB id: 1 thx $)$ and glutaredoxin from pig (PDB id: $1 \mathrm{kte}$ ) share $9.5 \%$ sequence identity, while spinach (PDB id: 1f9m) and Escherichia coli (PDB id: 1fov) thioredoxins share only $9.8 \%$ sequence identity. This conservation is attributed to the functional role of the disulphide bond for the catalysis of thiol-disulphide exchange reactions required for many functions including electron and proton transport to essential enzymes like ribonucleotide reductase, for the formation 


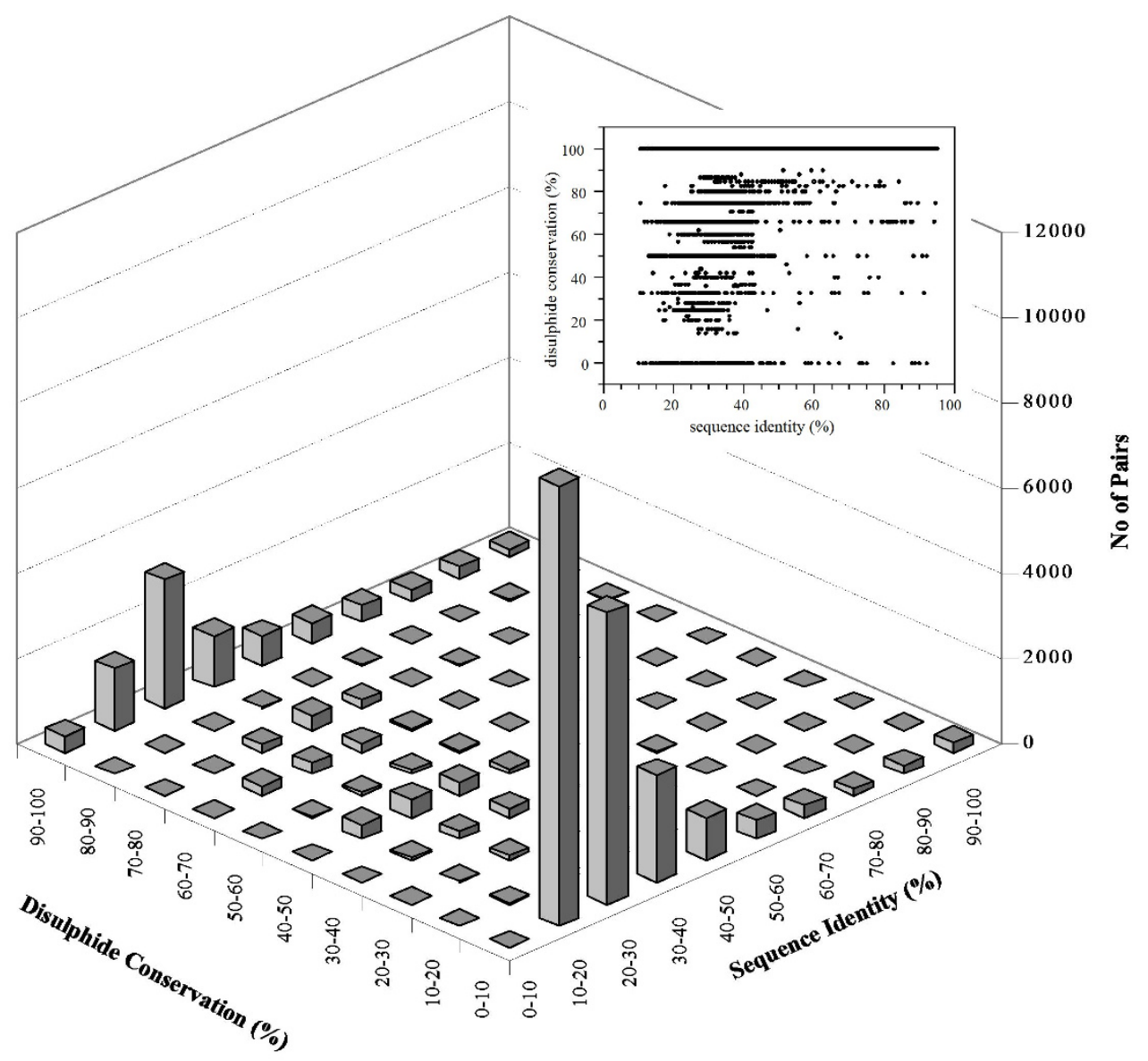

\section{Figure 4}

Disulphide bond conservation plotted against sequence identity for all the pairwise comparisons among the members of disulphide bond containing SCOP structural families. A total of 34,752 pairwise comparisons are made between members of the 300 families analysed. A two-dimensional scatter plot is also illustrated in the inset.

of disulphides in protein folding and for general regulation of protein function by thiol-redox control. On the other hand, the density, distribution and connectivity patterns of disulphide bonds in many small disulphide-rich proteins impart regularities to their structures and play a major role in determining their fold even in the absence of clear sequence similarity. The three disulphide bonds in the family of small Kunitz-type inhibitors and BPTI-like proteins (SCOP family code: g.8.1.1) are highly conserved with their sequence similarity in the range of 28.6 to 95.6\%. The members of this family, along with soft tick anticoagulant proteins (SCOP family code: g.8.1.2) classified under BPTI-like superfamily in SCOP, can be clustered into a single group based on the disulphide bonding pattern[28] while the sequence similarity across these families is as low as less than $20 \%$.
In SCOP classification, sequence identity is not a lone criterion to cluster families of proteins[42]. When established by similar functions and structures, proteins are grouped together into same families even if their sequence identities are below 30\%. The members of plant defensin family (SCOP family code: g.3.7.5) share four highly conserved disulphide bonds with a sequence similarity sometimes as low as $13.3 \%$. Similarly, ATX Ia (a neurotoxin) and BDS-I (an antiviral protein) do not share any observable sequence similarity but share three highly conserved disulphide bonds[70].

\section{Interactions among the substituted residues}

When a disulphide is not conserved, the local interactions among the substituted residues in the homologues lacking disulphide evoke some interest. Hence, to understand the functional implications of this non-conservation, we 
Table 3: Reasons for non-conservation of disulphide bonds between homologous proteins that share high sequence similarity.

\begin{tabular}{|c|c|c|c|c|c|c|c|}
\hline \multirow[t]{2}{*}{ SCOP Family } & \multicolumn{2}{|c|}{ PDB code of Proteins } & \multirow{2}{*}{$\begin{array}{l}\text { Cysteine } \\
\text { positions of } \\
\text { the SS bond }\end{array}$} & \multirow{2}{*}{$\begin{array}{l}\text { Sequence } \\
\text { Similarity } \\
\%\end{array}$} & \multirow{2}{*}{$\begin{array}{l}\text { Reason for non- } \\
\text { conservation }\end{array}$} & \multirow[t]{2}{*}{ Comment } & \multirow[t]{2}{*}{ Ref } \\
\hline & with SS & without SS & & & & & \\
\hline $\begin{array}{l}\text { Rubisco, large subunit } \\
\text { (c.I.I4.I) }\end{array}$ & 3rub & 8ruc & $172-192$ & 94.7 & $\begin{array}{l}\text { Crystallographic } \\
\text { artifact }\end{array}$ & $\begin{array}{l}\text { Not clear if functionally significant or formed during crystallization } \\
\text { period }\end{array}$ & [83] \\
\hline $\begin{array}{l}\text { Purple acid phosphatase } \\
\text { (d.159.1.I) }\end{array}$ & lute & Iqhw & $\mid 63-221$ & 84.7 & $\begin{array}{l}\text { Crystallographic } \\
\text { artifact }\end{array}$ & No indication crosslink in electron density map & [84] \\
\hline VHS domain (a.I I8.9.2) & lujk & Ijuq & $33-76$ & 72.7 & $\begin{array}{l}\text { Crystallographic } \\
\text { artifact }\end{array}$ & Not favorable to accommodate cross link & - \\
\hline $\begin{array}{l}\text { DnaQ-like 3'-5' exonuclase } \\
\text { (c.55.3.5) }\end{array}$ & Inoy & $\operatorname{lih} 7$ & $4 \mid-55$ & 63.9 & $\begin{array}{l}\text { Crystallographic } \\
\text { artifact }\end{array}$ & Not favorable to accommodate cross link & - \\
\hline Transferrin (c.94.1.2) & - & $\begin{array}{l}\text { Igv8 } \\
2 \mathrm{tmp}\end{array}$ & - & - & $\begin{array}{l}\text { Fragments of } \\
\text { domains }\end{array}$ & $\begin{array}{l}\text { I gv8 - fragment of } \mathrm{N} \text {-terminal domain of the intact protein, } \\
\text { ovotransferrin } \\
2 \text { tmp - only I-I } 22 \text { residues of } \mathrm{N} \text {-terminal domain of TIMP }\end{array}$ & [85] \\
\hline Annexin (a.65.1.1) & - & Iscf & - & - & $\begin{array}{l}\text { Fragments of } \\
\text { domains }\end{array}$ & Partial structure & - \\
\hline G proteins (c.37.I.8) & - & $\begin{array}{l}\text { Iryh } \\
\text { ImhI } \\
\text { Idsb }\end{array}$ & - & - & $\begin{array}{l}\text { Fragments of } \\
\text { domains }\end{array}$ & Partial structure & - \\
\hline $\begin{array}{l}\text { Glutathione S-transferase, } \\
\text { N-terminal domain } \\
\text { (c.47.1.5) }\end{array}$ & $\mathrm{IkOm}$ & Irk4 & $24-59$ & 98.6 & Structural transition & $\begin{array}{l}\text { A typical glutathione S-transferase but with a glutaredoxin-like } \\
\text { active site. Disulphide bond facilitates a redox-controlled } \\
\text { structural transition from monomeric to dimeric state }\end{array}$ & [49] \\
\hline Prion-like (d.6.I.I) & $\mathrm{li} 4 \mathrm{~m}$ & luw3 & $|79-2| 4$ & 91.2 & Structural transition & $\begin{array}{l}\text { Rearrangement of disulphide bonds helps in conformationallly } \\
\text { altering the prion protein to pathogenic oligomeric form. }\end{array}$ & [50] \\
\hline $\begin{array}{l}\text { Alpha-macroglobulin } \\
\text { receptor domain (b.2.4.I) }\end{array}$ & layo/Ibv8 & ledy & $17-132$ & 66.2 & Structural transition & $\begin{array}{l}\text { Major conformational differences between human/bovine and rat } \\
\text { RBD }\end{array}$ & [60] \\
\hline Papain-like (d.3.I.I) & Iqdq & $3 \mathrm{pbh}$ & $148-252$ & 88.9 & $\begin{array}{l}\text { Stabilization of local } \\
\text { structure }\end{array}$ & $\begin{array}{l}\text { Disulphide bond increases the conformational flexibility of the } \\
\text { occluding loop, although the conformational stability of the overall } \\
\text { structure is little affected. }\end{array}$ & [54] \\
\hline Parvalbumin (a.39.1.4) & la75 & Ibu3 & $11-33$ & 88.7 & $\begin{array}{l}\text { Stabilization of local } \\
\text { structure }\end{array}$ & $\begin{array}{l}\text { This disulphide bond is unique to Whiting parvalbumin and } \\
\text { stabilizes the two helical hairpin although the conformational } \\
\text { stability of the overall structure is little affected. }\end{array}$ & - \\
\hline $\begin{array}{l}\text { Dipeptidyl peptidase IVI } \\
\text { CD26, N-terminal domain } \\
\text { (b.70.3.I) }\end{array}$ & Inu6 & Iorv & $328-339$ & 85.7 & Increased activity & $\begin{array}{l}\text { Adenosine deaminase (ADA) binds stronger to the disulphide } \\
\text { bonded human protein than in porcine. }\end{array}$ & {$[51]$} \\
\hline Ricin B-like (b.42.2.I) & 2aai & $\begin{array}{l}\text { Im } 2 \mathrm{t} \\
\text { Ionk }\end{array}$ & $20-39$ & 72.6 & Increased activity & Reduced cytotoxicity in mistle toe lectin & [52] \\
\hline $\begin{array}{l}\text { Xylanase/endoglucanase II/ } \\
\text { I2 (b.29.I.II) }\end{array}$ & lyna & Ixnd & $110-154$ & 59.5 & Increased activity & $\begin{array}{l}\text { Increased thermostability due to compounding effect of disulphide } \\
\text { bond with increase in the density of charged particles }\end{array}$ & [53] \\
\hline $\begin{array}{l}\text { Mycobacterial antigens } \\
\text { (c.69.1.3) }\end{array}$ & IfOn & Idqz & $87-92$ & 72.1 & Unassigned role & $\begin{array}{l}\text { Not obvious from the structural differences if the antigens have } \\
\text { different biological roles }\end{array}$ & [55] \\
\hline $\begin{array}{l}\text { Quinoprotein alcohol } \\
\text { dehydrogenase-like } \\
\text { (b.70.I.I) }\end{array}$ & $\lg 72$ & $\begin{array}{l}\text { Ikb0 } \\
\text { lkb9 }\end{array}$ & $144-167$ & 33.2 & Unassigned role & No structural or functional role assigned & [56] \\
\hline Subtilases (c.4I.I.I) & Idbi & Ithm & $137-139$ & 61.6 & Unassigned role & $\begin{array}{l}\mathrm{C}-\mathrm{X}-\mathrm{C} \text { disulphide bridge is hypothesised to enhance the } \\
\text { thermaostability }\end{array}$ & [57] \\
\hline Hemorrhagin (d.92.1.9) & Ibud & 4aig & $157-164$ & 49.2 & Unassigned role & $\begin{array}{l}\text { No direct evidence if the variable disulphide bridges in the C- } \\
\text { terminal subdomain hemorrhagin family of enzymes lead to } \\
\text { differences in their hemorrhagic activity. }\end{array}$ & [58] \\
\hline
\end{tabular}


evaluated the possible interactions among the substituted residues lying in topologically conserved regions of the family of proteins.

In all, we have observed 7064 substitutions out of which, as expected, $87 \%$ are accounting for low or poorly conserved disulphide bonds. In about $25 \%$ of these poorly conserved disulphides, the bonded cysteines are aligned with gaps in the alignment that was derived from structural superimposition. Another 35\% have at least one cysteine residues aligned with a gap. These depict situations where disulphides do not share topologically equivalent counterparts. Otherwise, in only $3 \%$ of the situations, both cysteines are aligned with residues in topologically conserved regions. These substituted residues can undergo, at best, non-covalent interactions and rarely covalent interactions (e.g. transglutamination), if any. Although in principle all interactions are electrostatic in nature, hydrogen bonding, hydrophobic interactions, aromatic and aliphatic-aromatic interactions were investigated separately.

A total of 54 distinct disulphides distributed in 43 families featured 213 topologically equivalent substitutions. These substitutions were studied with respect to the conservation status (highly or poorly conserved) of their native distinct disulphide. These results are featured in additional file 1 . In order to avoid any bias inherent to our dataset, we further analysed the interactions at $40 \%$ sequence identity. The same general trend was observed.

It appears from our data that, in cases where the disulphides are generally highly conserved in the family (23 out of 54 disulphides), in the members that lack these disulphide bonds, there is a clear preference for hydrophobic residues among substituted residues (Additional file 1). Several of these disulphide substitutions cases are well documented in the literature. Examples are the PhePhe interaction that stabilises the monomers of $\alpha_{2} \mathrm{M}$ rat receptor binding domain (PDB id: 1edy) by bringing their amino- and carboxy-termini together[71]; the $\pi$-conjugation type interaction between Tyr 25 and Ala106 residues in the anion binding site of YBHB from $E$. coli hence tightening the loops GXG and CR2 (a conserved motif) to each other[72], the aromatic-alphatic interaction between Trp48 and Leu-65 in BphF, a Rieske-type ferredoxin associated with biphenyl dioxygenase (PDB id: $\underline{1 \mathrm{fat}}$ ), which importantly contributes to lower the redox midpoint potential of the protein by approximately $500 \mathrm{mV}[73]$.

On the other hand, the substitutions at positions of poorly conserved disulphide bonds ( 31 out of 54 distinct disulphides) correspond to residues that are not congenial for favourable inter-residue interactions (Additional file 1). A typical case is illustrated by adenylosuccinate lyase
(SCOP family code: a.127.1.1) from the three disulphide bonded hyperthermophilic Pyrobaculum aerophilum and its homologue from Thermotoga maritima where the absence of three disulphides is compensated through other sequence and structural features within the vicinity of the disulphide bond of these proteins but not directly by the substituted residues[59]. Where a disulphide bond is absent, interactions among stabilization center residues, clusters of residues in protein contact maps where an accumulation of long range interactions are observed $[74,75]$, also compensate for the absence of disulphide. Other typical cases are further documented in Additional files.

\section{Backbone conformation and residue length separating the half-cystines and conservation}

Figure 5 represents the secondary structure at the two halfcystines of disulphide bonds in the present data set. It can be seen that the disulphide bonded cysteines correspond predominantly to coil-coil and coil-strand connectivities. Figure. 5 also depicts the secondary structure preferential distribution of half-cystines in different fold classes. It can be seen that the disulphide secondary structure connectivity preference is not biased by the small proteins which is the most populated protein class with a total of 1385 disulphides and by the fact that small proteins have a large preference $(77 \%)$ for half-cystines to connect a coil region.

It is expected that local structures of the half-cystine containing regions would be topologically conserved in homologous proteins along with the conservation of the disulphide bond itself. As previously shown (cf supra), in about $70 \%$ of the disulphide bonds, at least one of the two half-cystines is structurally conserved. This is rather interesting since more than $68 \%$ of disulphide bonds in our data set occur in loop regions or connect regular secondary structures with coiled regions. Hence, the highly variable nature of loops/coils did not deter the disulphide bond conservation, strongly suggesting their role in structural stabilization (Figure. 5). On the other hand, in almost $80 \%$ of the non-conserved disulphide bonds, the secondary structure status of at least one half-cystine is in coil state. This suggests that disulphide bonds bring rigidity to such structurally variable regions in homologous proteins. In general, neither highly conserved nor low conserved disulphides display a preference for a particular local backbone conformation.

An overwhelming majority (97\%) of the disulphide bonds studied in our dataset have their half-cystines separated by less then 50 residues. A subset of our initial data, culled at $40 \%$ sequence identity, has been analysed by dividing them into five groups with increasing loop sizes of 50. Figure. 6 shows the probabilities for these groups to 
be highly or poorly conserved, indicates clear trends. A majority of the poorly conserved disulphides (i.e. nearly $75 \%$ ) are non-local (> 100 residue separation) in the current analysis. Similarly, about $40 \%$ of non-local disulphides are poorly conserved and this drops to $20 \%$ in the case of local disulphides. Small proteins did not bias this observation since the data was analysed with and without the small proteins. Non-local disulphide bonds are less frequent and are not as much important as local disulphides which helps in kinetic folding by bringing together local regions of the protein [76].

\section{Solvent accessibility of disulphides and conservation}

Since the relationship between extent of conservation of disulphides and their solvent accessibility is not yet known, we performed a survey of the solvent burial of disulphides in our dataset and integrated the information on extent of disulphide conservation. In this analysis, disulphide bond burial has been defined as follows: when both half-cystines are buried, the disulphide has been considered as 'buried'; when one half-cystine is buried and the other solvent exposed, the disulphide is considered as 'partially exposed'; when both half-cystines are sol-

a)

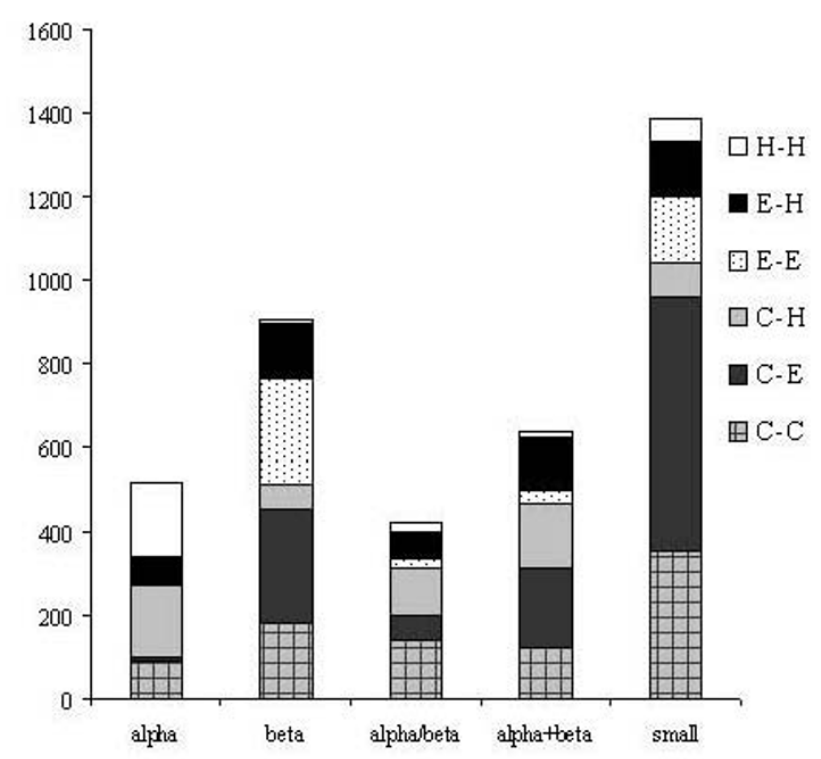

vent accessible, such disulphides are considered as 'exposed'.

From Figure 7 and further analysis of the data it can be inferred that overall, buried as well as fully exposed disulphides share the same tendency (66-72\%) for conservation. It should be noted that nearly $44 \%$ of the exposed half-cystines belong to small proteins, which can be reasoned by the lack of definite hydrophobic core as in globular proteins, thus emphasising their importance in maintaining the fold through the structural scaffold. However, it is noteworthy that buried as well as exposed disulphides are also vulnerable to mutations with as much as $13 \%$ and $19 \%$ respectively that are low conserved. Our results hence seem to establish that there is no apparent preference between high conservation and the solvent accessibility status of disulphides, i.e. high conservation of a specific disulphide can be an important feature of the disulphide irrespective of whether it is buried or exposed. Our results also suggest that there is no preference for poorly conserved disulphides to be exposed or buried. Similarly, our data shows that disulphide exposure would not necessarily be associated with low conservation.

b)

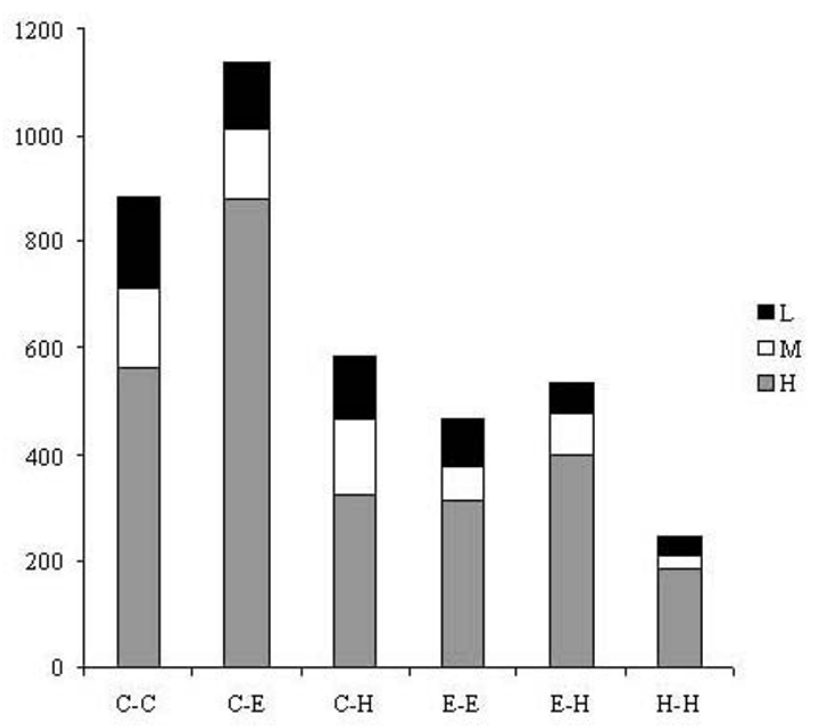

Figure 5

Fold distribution of different backbone conformations of disulphide bonds: Alpha, beta, alpha/beta, alpha+beta, and small proteins are different SCOP fold classes; $\mathrm{C} / \mathrm{E} / \mathrm{H}$ are coil/strand/helical conformations of the half-cystines. a) Backbone conformational preferences of disulphide bonds in different fold classes, b) Distribution of disulphide bond backbone conformations into different conservation classes; L-low or poorly conserved, M-medium conserved, and H-highly conserved. 
However, when a distinction between small proteins and bigger proteins is made (Figure. 7), a higher proportion of partially or fully exposed disulphides are poorly or medium conserved. Though very slight, there is a general tendency in bigger proteins for exposed disulphides to be less conserved than buried disulphides. This is particularly clear in $\alpha / \beta$ proteins. From $58 \alpha / \beta$ protein families containing 427 disulphides about $70 \%$ of the partially or fully exposed disulphides are low or medium conserved. On the other hand, high conservation of disulphides is common in small proteins and it is independent of the status of their disulphide solvent accessibilities (Figure. 7, also see Figure. 2 for distribution of disulphide bonds in different fold classes).

\section{Stereochemistry versus conservation of disulphide bonds} Analysis of disulphide bridge stereochemistry might give clues if there exists a relation between geometry and conservation of disulphide bonds. The bridge stereochemical parameters are calculated for all the native disulphide bonds in the database and are graded according to the criteria defined by Sowdhamini and co-workers [26,77]. A native disulphide bond, irrespective of its level of conservation, tends to appear in ideal stereochemistry (data not shown).

To perform this analysis, we selected a subset of protein structural data of high quality (571 disulphide bonds from 172 proteins) of our original data, with a crystallo-

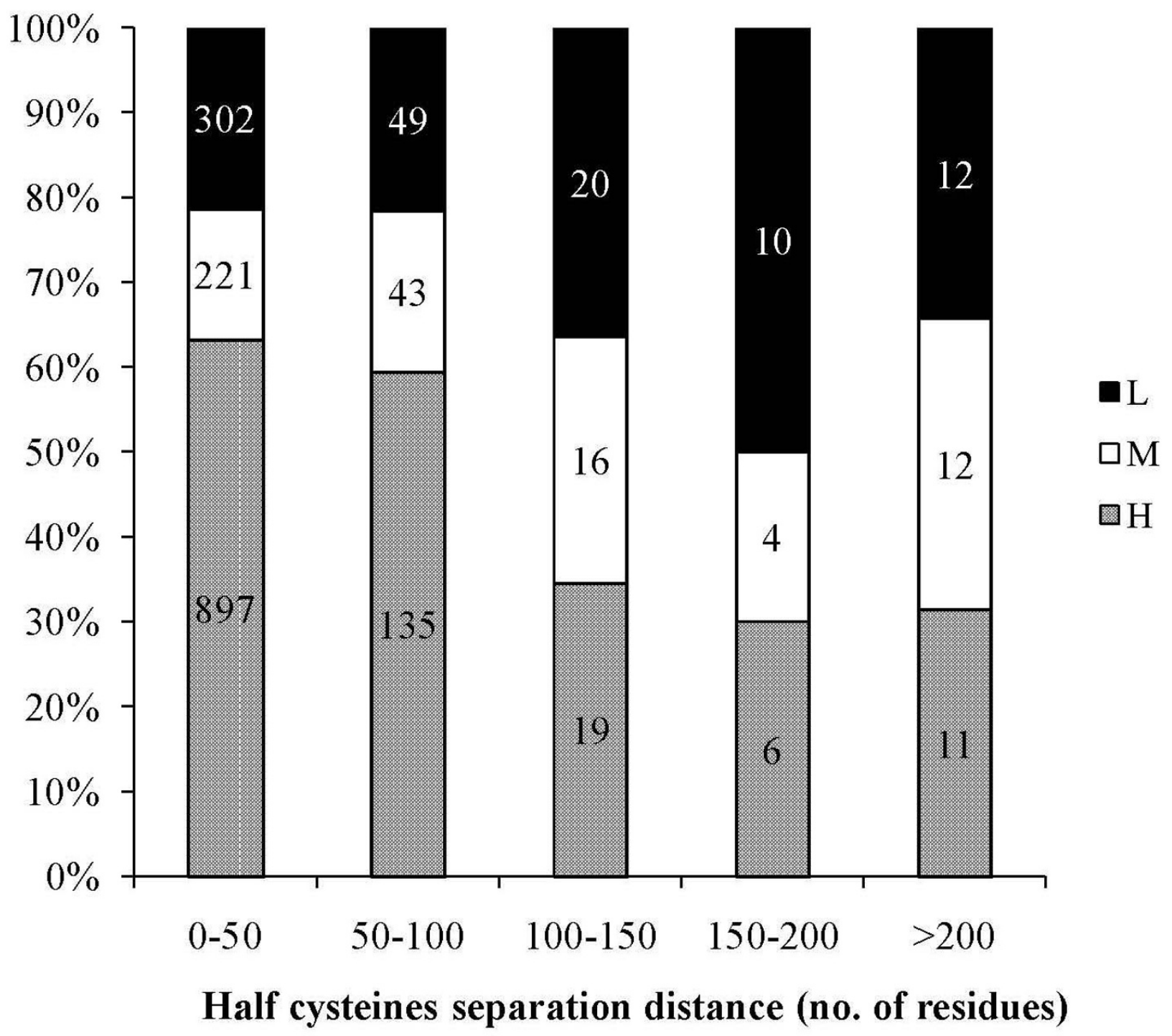

Figure 6

Conservation status ( $H, M$ or $L)$ of disulphide bonds as a function of half-cystine separation distance. 
graphic resolution better than $2 \AA$ and R-factor 0.25 and sequence identity between homologues less than $25 \%$.

The distribution of $\mathrm{C} \alpha$ and $\mathrm{C} \beta$ distances in the well and poorly conserved classes of disulphide bonds are very similar and did not show any significant deviation from ideal values (data not shown). Similarly, the SS bond dihedral angle showed similar distribution patterns. Although poorly conserved disulphide bonds are not necessarily non-local, as shown in previous section, several non-local disulphide bonds are poorly conserved. It would hence be of interest to see if the poorly conserved non-local disulphide bonds are strained or show deviations. Interestingly, contrary to the observation of Thornton[2] based on limited data that local disulphide bonds tend to be right handed, we observed a marginal preference to be left handed in highly conserved disulphide bonds. Low or poorly conserved disulphide bonds distributed equally in

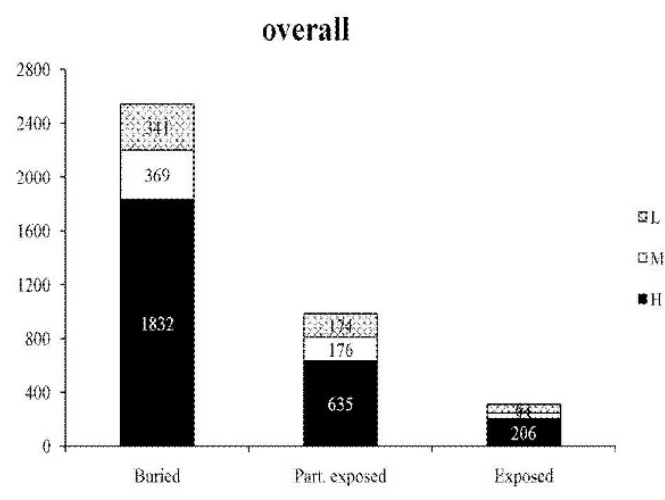

$\beta$ proteins

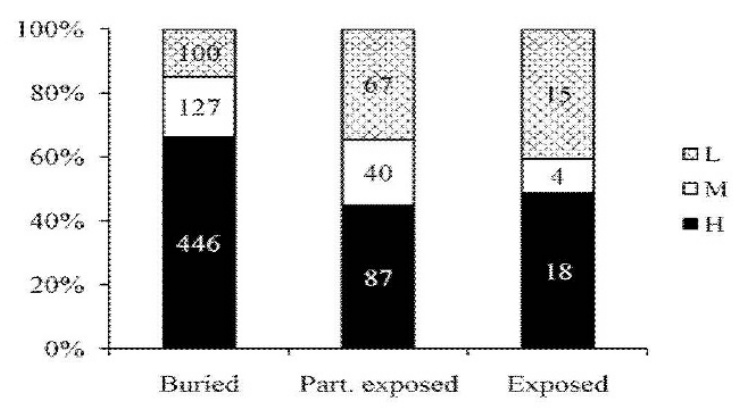

$\alpha+\beta$ proteins

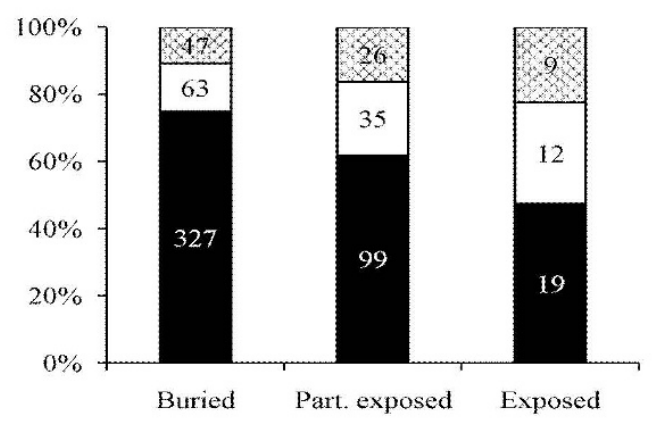

\section{$\alpha$ proteins}

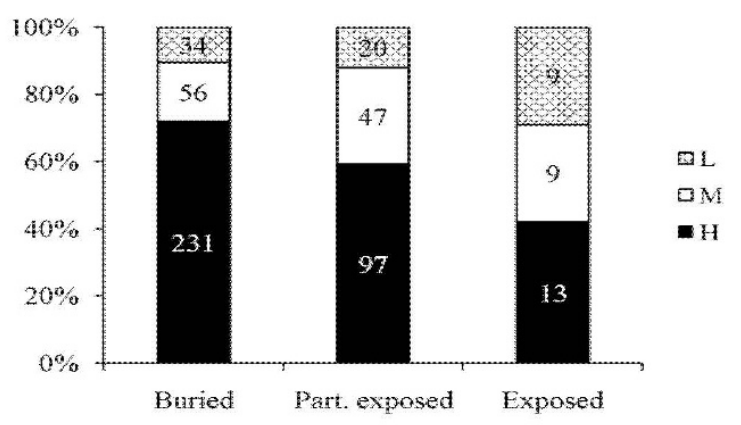

\section{$\alpha / \beta$ proteins}

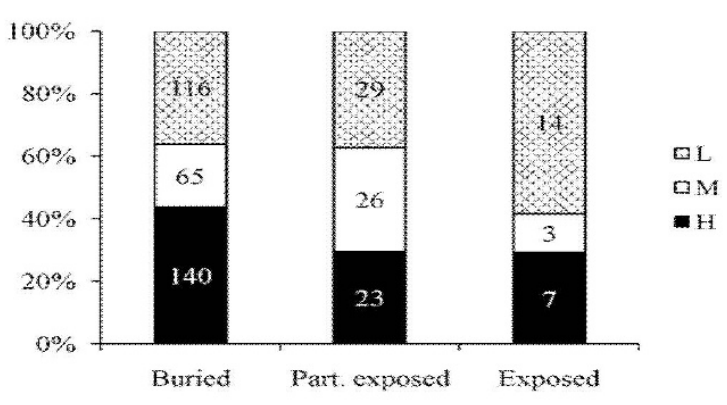

\section{small proteins}

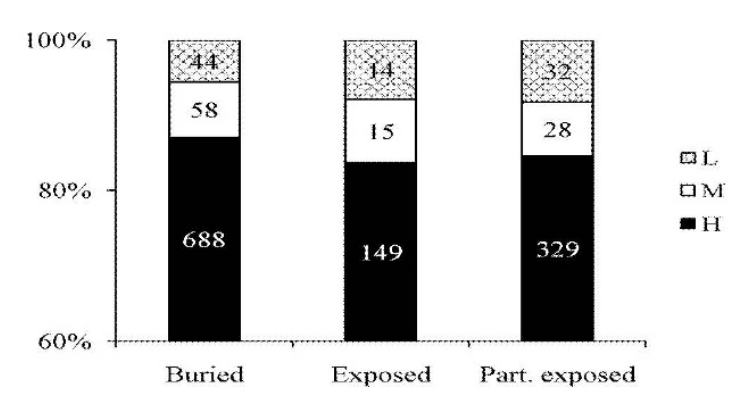

\section{Figure 7}

Analysis of solvent accessibility (buried, partially exposed and exposed) of disulphide bonds and their conservation status $(H, M$ and $L)$ with regard to the SCOP classification of proteins. 
left handed and right handed conformations. However, non-local disulphide bonds, although sparse, indeed showed slightly higher preference to be left-handed (Figure. 8).

The distribution of all the bridge torsion angles ( $\chi$ ss, $\chi 1$, $\chi 2$ ) of both highly and poorly conserved disulphide bonds were plotted and the respective histograms were very similar and also showed close similarity with previous studies (data not shown)[78]. Stereochemical evaluation necessitates studies of the specific families, since stereochemistry can be influenced by the local sequence variations of the homologues.

Overall, our results suggest that extent of conservation of a disulphide is independent of the conformation of the bridge.

\section{Variations of disulphides at family and superfamily level}

As stated in the previous sections, non-conservation of distinct disulphide bonds within homologous families is a common feature and we analysed its biological implications through several examples. Such an analysis can be extended by investigating the conservation and structural variations of disulphides between members within a homologous family and across families in a superfamily (see Additional file 2 for a discussion on limited number of examples and Additional files 3, 4, and 5 for associated figures). This analysis, though limited to a superfamily, illustrates non-conserved disulphide bonds across superfamily members have limited effect on their common fold, but could be associated with recruitment of new structural features.

\section{Discussion}

The purpose of this study is to examine the question of the extent of conservation of disulphide bonds in homologous proteins. The whole study is performed from a structural point of view so that we do not have to compensate for the quality of alignments nor make any assumptions on disulphide bond connectivity after including sequence homologues. For long, disulphide bonds are seen as conserved features like enzyme active sites, and often used to draw relations between distant relatives. There has been no general or systematic study previously on the structural conservation of disulphide bonds amongst a large number of protein domain families, although several protein specific studies highlighted the presence or absence of an extra disulphide bond in close homologues. The availability of classified information in terms of homologous protein families with sufficient number of structural entries has prompted us to carry out a large-scale analysis to quantify the extent of conservation of disulphide bonds.
The distribution of these mutations of disulphides is widespread in all structural classes and grading the extent of conservation enabled in quickly identifying the variable crosslinks. We found that the discriminative ability of such a large-scale analysis is high, helps in quickly identifying highly conserved disulphide bonds in homologues, which might play a crucial role in protein folding or maintaining the fold. Almost $60 \%$ of substitutions of poorly conserved disulphides in our dataset are gaps, suggesting that the impact of such disulphide bonds on protein folding kinetics could possibly be limited. For example, disulphide bonds in or near the folding nucleus enhance folding and are usually conserved in related proteins. Disulphides outside the folding nucleus nevertheless have different functions like in extracellular proteases (Rossmann fold containing proteins) where they protect a cell against errors in translocation and undesirable protease activity while they are in cytoplasm[30].

Protein structures diverge linearly, although at a much lower rate, with the sequence variability in homologous proteins $[79,80]$. However, several families of proteins, involving distant relatives, withstand such an evolutionary pressure and show minimal or no correlation between sequence and structure variation. While this could be due to the strong functional constraints like catalytic site conformation[81], disulphide bonds stabilizing the protein core form the structural constraints[79]. Our approach to study disulphide conservation holds the information on structural evolution of homologous proteins since it inherently encompasses the sequence-structure dependence. Hence, the invariance of disulphide bond conservation with the sequence divergence (see Figure. 4) can be reasoned to selection pressure, preserving these bonds due to their strong structural/functional role. We also found only in less than $15 \%$ single disulphide bonded families the crosslink is highly conserved. Similarly, abundance of disulphide (number of disulphides in a protein) has little effect on conservation. This suggests there are other factors that influence the sequence-structure relationship and there is no relation between the number of disulphide bonds and their conservation. Hence, conservation of disulphides can be reasonably explained by the important implications of disulphides in the structures and/or functions of proteins.

Disulphide bonds playing a functional role are usually highly conserved, where as disulphides in structurally similar but functionally different proteins are poorly conserved. In fact, disulphides probably have varying degree of conservation among homologous proteins depending upon if a given disulphide have structural or folding or functional or regulatory role. Sometimes a given disulphide might play more than one role. In practice such an analysis is very difficult to perform as detailed knowledge 


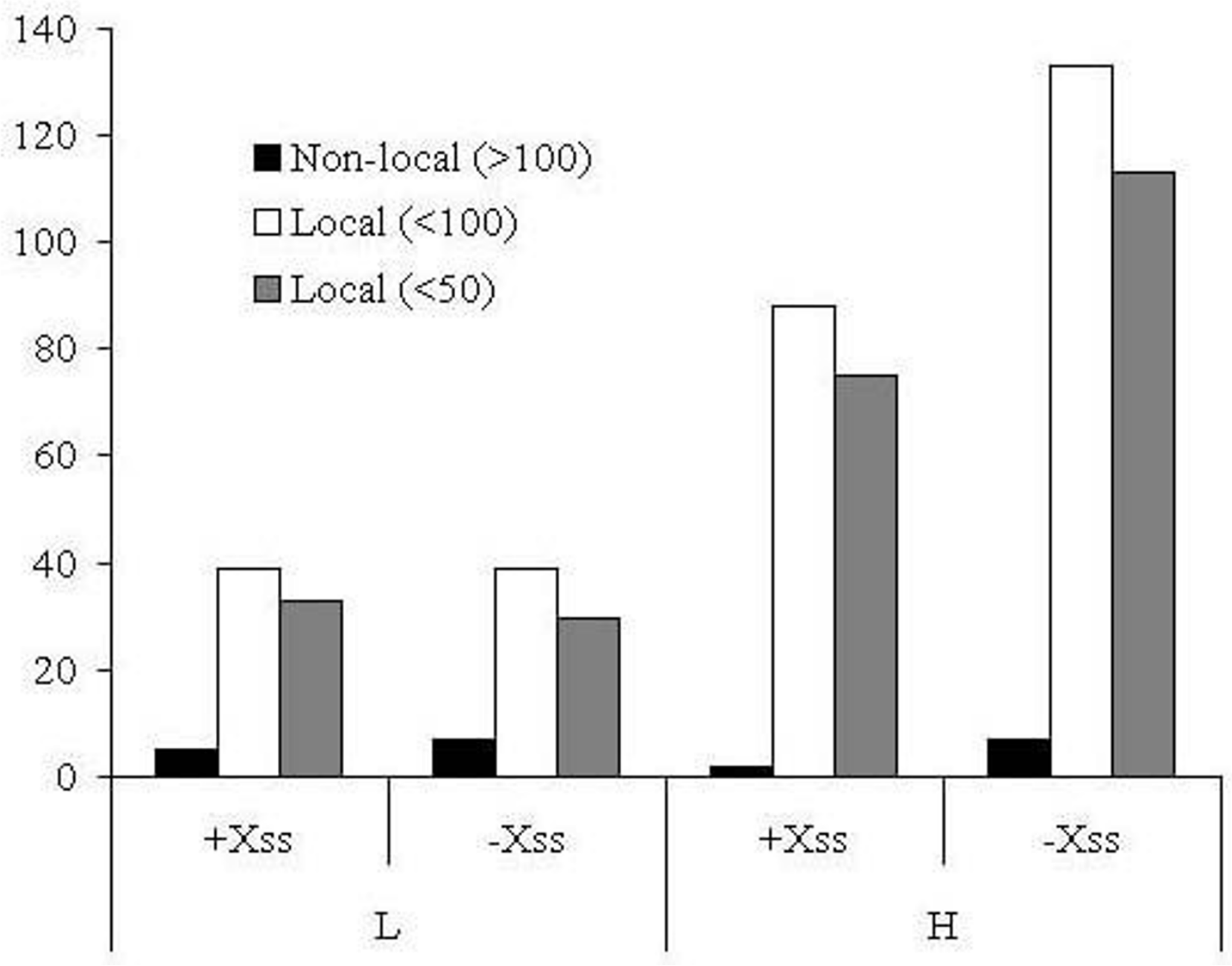

Figure 8

Preferences for handedness of disulphide bonds in highly $(H)$ and poorly $(L)$ conserved disulphide bonds with respect to their Chi angle of the SS bond. -ve left-handed; +ve right-handed.

on structural/folding/functional/regulatory roles of most disulphides in most proteins is not available.

We show that non-conservation of disulphides within protein families can be associated with substitutions displaying favourable interactions or with other subtle structural changes that compensate the loss of covalent link in a homologue compared to another. Disulphide bond mutation not always changes the basic structure of the protein[2], but might affect the activity of the protein without significantly changing the structure[82,83], sometimes even without altering the function[39,84]. Our work also shows that non-conserved disulphides are often associated with variable structural features that were recruited during evolution and this recruitment is generally associated with differentiation or specialisation of protein function. One of the major implications of the non-conservation of disulphides within homologous families is that it confers plasticity to disulphide connectivity patterns. Our study clearly re-establishes that this plasticity is correlated to structural or functional constraints. Conserved disulphides result in conservation of their patterns, although with a few exceptions (conserved topology but not the connectivity pattern). The common or mixed ancestry of diverse proteins can be recognized by conservation of disulphide bonds $[28,29,70]$ even when most of the surrounding residues have been replaced[85]. Attempts were made to map the patterns of paired natural cysteine mutations[41]. It has been shown that cystine mutations are coordinated and results in the loss of the disulphide bond[2] and such a feature can be used to identify the connectivity of cystines in multiple disulphide bonded families[41]. 
It has been noted that remote homology detection using disulphide bond connectivity, is severely limited not only by the requirement of their natural occurrence $[28,70]$, but also by the lack of information on how far one can relax the size of the disulphide loops during searches. The availability of a library of disulphide profiles in the future might facilitate associating proteins with new connectivity and for the rational design of site-directed mutagenesis experiments.

We clearly demonstrate here that conservation of disulphides is a family-specific feature and is neither correlated to solvent accessibility status nor to backbone conformation. While disulphide bonds are common in all fold classes, their preference for stabilizing strands and loops has concentrated their presence in beta-strand class and in small proteins. Also the extracellular or secreted proteins, where disulphide bonds almost exclusively appear with some exceptions $[86,87]$, usually adopt $\beta$ and $\alpha+\beta$ type structures having more beta structures stabilized in part by medium- and long-range non-covalent interactions apart from disulphide bonds $[74,88,89]$. Hence the secondary structural preferences of extracellular or secreted proteins and streochemical restraints at the backbone regions where disulphide bonds are accommodated appear to be inter-related. The evolutionary plasticity of loop regions is greater than that of protein core [79] and this would explain the greater crosslink frequency in the loop regions of homologous proteins and which helps in maintaining the fold.

Interestingly, our work demonstrates that there is no clear correlation between sequence similarity and degree of disulphide conservation. It is shown that high sequence similarity does not guarantee disulphide bond conservation. Genuine cases where highly similar proteins do not share common disulphides are documented. To our knowledge, this is the first documented study that focuses on such a relationship, thus highlighting the singularity of the evolutionary dynamics of disulphides bridges in homologous protein structures. The idea of analysing disulphide bond conservation in protein families has recently been implemented into a web facility freely accessible at http://bioinformatics.univ-reunion.fr/analycys/ [90].

\section{Methods \\ Dataset}

The release 1.67 of SCOP [42] containing 2630 families with over 65122 domains has been used in the present analysis. The SCOP database, created by manual inspection and abetted by a battery of automated methods, broadly classifies all proteins in to five major classes, alpha, beta, alpha/beta, alpha+beta and small proteins. Small proteins are usually with less than 70 residues and are rich in disulphide bonds. Disulphide bond definitions of the domains are extracted from the SSBOND record of the corresponding PDB [91] files. Only intra-domain disulphide bonds are considered in the present study.

Of these, 580 families have at least one member with disulphide bond. Ignoring multidomain and membrane classes resulted in 557 families with at least one member with a disulphide bond. The dataset is pruned to remove redundancy (mutant and identical protein entries) rather than close sequence homologues. Filtering the initial dataset at 95\% sequence identity and a resolution better than $2.5 \AA$ has resulted in 300 families containing at least two members in each family. For proteins with NMR derived structures, the first model in the ensemble of structures was considered for superimposition.

When we tried to enrich the dataset to include homologous proteins of yet unknown structure, we encountered two problems: Firstly, in the case of distant, yet, definite homologues it is difficult to be confident of good quality of alignment with the homologues as structure is unavailable. Second problem which is even more serious is the non-availability of disulphide bond connectivity established by experimental methods for several sequences. If cysteines involved in disulphide formation in a protein are conserved in a homologue of unknown structure, one can be fairly confident about such disulphide connectivity in the homologue with yet unknown structure. However, if the cysteines occur in unconserved positions, especially in insertions, in the protein of unknown structure then the disulphide connectivity information for the protein of unknown structure is incomplete. Due to these problems we confined our analysis only to proteins of known 3-D structure.

\section{Cellular localisation of proteins in the dataset}

Three prediction methods (TargetP[92], PSORT[93] and SubLoc[94]) and literature curation (Additional file 6) was performed, where possible, on the full-length sequence of the proteins in the entire dataset. Where all three or two out of three methods predict a protein to be cytoplasmic, we have examined the residues at the site of disulphide bond positions in the corresponding family (see Additional files 7 and 8).

\section{Structural superimposition}

Structure-based sequence alignments in these SCOP families were derived using STAMP package[95]. STAMP aligns a set of homologous protein structures and generates a structure-based multiple sequence alignment among others. Topologically equivalent regions usually correspond to conserved secondary structures within a family of structures under comparison. Topologically equivalent regions from the multiple structural alignments are derived by a method of Argos and Rossman, implemented internally in the structural alignment pro- 


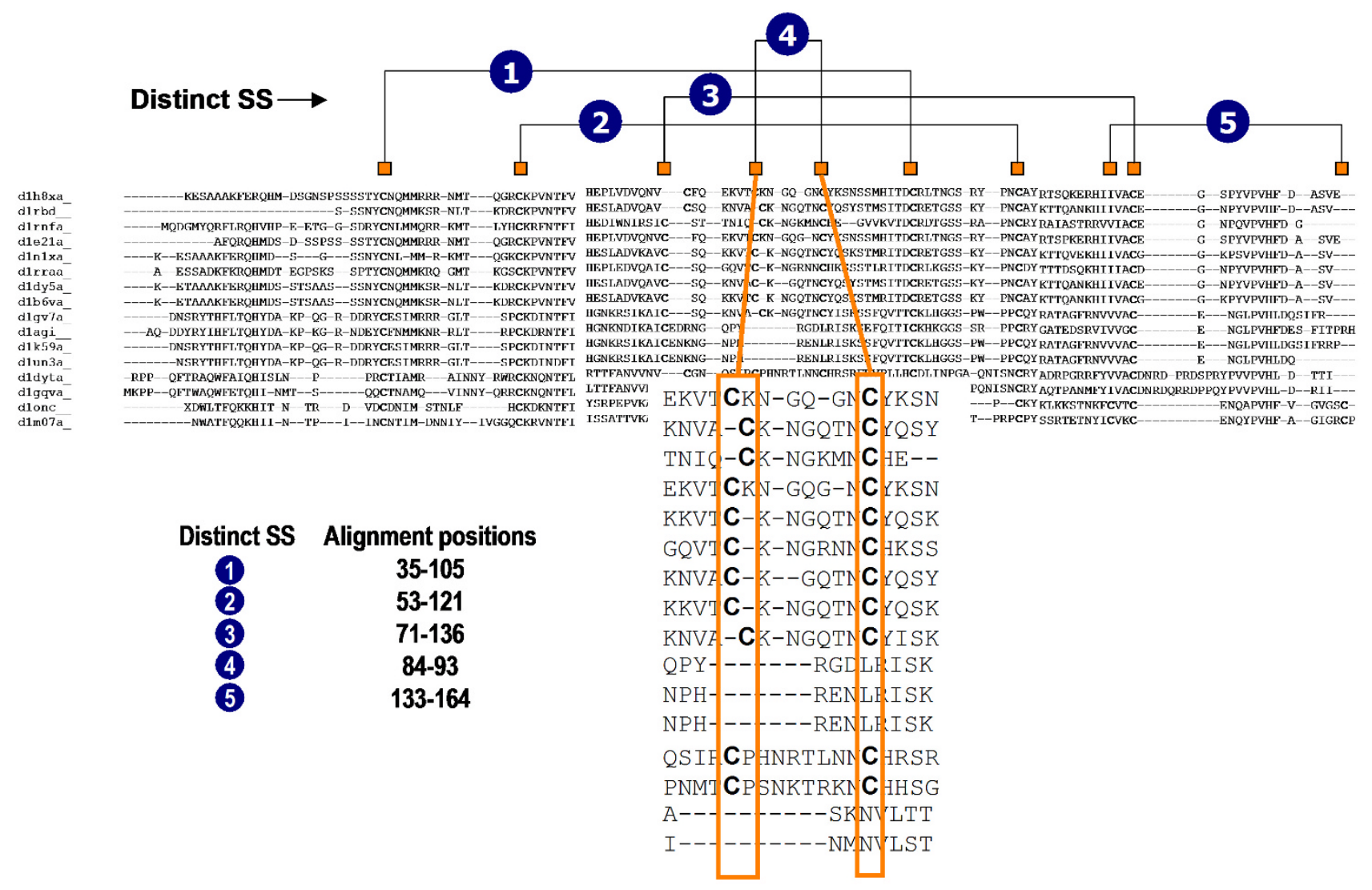

Figure 9

Illustration of distinct disulphide bond annotation in Ribonuclease A-like [SCOP family code: d.5.I.I] family of proteins. The structural alignment contains sixteen members. Marking of the disulphide bond positions on the structure based multiple sequence alignment has resulted in five distinct structural positions. However the maximum number of disulphides in a family member is only four, while the minimum is three. Each distinct disulphide bond is represented with approximate positions of their cysteines in the alignment. In the zoom out of the alignment region of the 4th distinct disulphide bond note the first cysteine is not in equivalent position, nevertheless it is conserved. Applying relaxation brings this disulphide bond into conservation. Also notice that this disulphide bond is not conserved in all the members.

gram by calculating the probability of residue structural equivalencies[96]. The block of multiple structure-based sequence alignment is considered as the alignment footprint of the family.

\section{Annotation of distinct disulphide bonds and grading of conservation in a family}

Native disulphide bond information has been extracted from the SSBOND record of PDB files of all protein structures. For every family in the data set, the positions of disulphide bonds in homologous members are marked on the structural alignment footprint of the family. The positions of the disulphide bonds in this footprint region are counted as distinct disulphide bonds in the family (Figure. 9). Such an annotation is referred in this paper as the 'disulphide profile' for the family and facilitates a global view of the connectivity pattern of all the disulphide bonds in a family of aligned proteins, irrespective of the presence or absence of a disulphide in the individual members. Once the distinct disulphide bonds in a family are identified, their conservation is calculated as a ratio between the number of proteins containing the distinct disulphide bond and the total number of proteins in the alignment. When there is no disulphide bond found in a member at a position, a relaxation (Figure. 9) has been applied by allowing an arbitrary shift of 4 residues on either side of the cysteine positions for counting the distinct disulphide bonds.

To understand the importance of the evolutionary variations of disulphide bonds in protein families, conservation is quantified and graded. A highly conserved disulphide bond is one that is present in more than $70 \%$ of the family members while medium and poorly conserved disulphide bonds are present in 30-70\% and less than $30 \%$ of the members of the family, respectively. 
Descriptors of structural environment of disulphide bonds Backbone conformation, solvent accessibility and residue depth are chosen to define the structural environment of the cysteines involved in disulphide bond formation. The backbone conformation and secondary structure assignment of half-cystine are derived using the program, DSSP[97]. However, a three state assignment is used instead of the default seven states. We grouped the eight DSSP labels into three categories: helix, H = 'HGI' (a-helix, 3-helix and p-helix), sheet, E = 'EB' and loop, C = 'TS' (turns and bends). Solvent accessibility has been calculated using the method of Lee and Richards[98] as implemented in the program NACCESS[99]. The relative accessibility of the half-cystine is its percentage solvent accessibility (SA) compared to Ala-Cys-Ala tripeptide. A half cystine with less than $10 \% \mathrm{SA}$ is considered buried, $10-25 \%$ is partially exposed and with more than $25 \%$ SA is considered exposed. Residue depth is calculated by using a program, DPX[100]. This program gives the depth of each atom in protein, defined as distance ( $\AA$ ) from the closest solvent accessible atom and calculates the mean residue depth. These depth values are critically dependant on local parameters like protein size, shape and structure. However, it is important to understand if there exists a correlation among solvent accessibility, conservation and depth of half-cystines. Hence the halfcystine in each protein are classified as core, intermediate or surface relative to the residue depth values of other residues in the protein.

\section{Abbreviations}

$\AA$ : Angstrom; DSSP: Define Secondary Structure of Proteins; DPX: Depth of each atom in Protein; NACCESS: Accessible Surface Area; NMR: Nuclear Magnetic Resonance; PDB: Protein Data Bank; SCOP: Structural Classification of Proteins; STAMP: Multiple Structure Superimposition Program; SSBOND: Disulphide Bond

\section{Authors' contributions}

RRT developed the algorithm, performed data analysis and provided draft of the manuscript. MM conducted literature survey on the sub-cellular localization. NS, RS and BO conceived and formulated the ideas and developed the strategies. FC designed the project, provided the overall plan and supervised the project.

\section{Additional material}

\section{Additional file 1}

Nature of side chains of of substituted residues topologically equivalent with cystines of a distinct disulphide bond in their respective families. For domains where the original disulphide bond is mutated, the topologically equivalent substituted residues are extracted to verify if the interaction is conserved.

Click here for file

[http://www.biomedcentral.com/content/supplementary/14726807-8-55-S1.doc]

\section{Additional file 2}

Evolutionary dynamics of disulphides at family and superfamily level. We extended our analysis by investigating the evolutionary dynamics of disulphides between members within a homologous family and also across families in a superfamily in order to provide further insights into the biological implications of conservation and non-conservation of disulphides. Click here for file

[http://www.biomedcentral.com/content/supplementary/14726807-8-55-S2.doc]

\section{Additional file 3}

Evolutionary dynamics of disulphides in the alpha/beta hydrolase actetylcholinesterase-like family (SCOP family code: c.69.1.1). Three highly superimposable members from this family are featured. In (a) is featured a representative (SCOP domain: d2bce_; PDB code: $\underline{2 b c e)}$ from the cholesterol esterase and carboxyesterase types which display two highly conserved disulphides. In (b) is featured a representative (SCOP domain: $d 1 d x 4 a \_$PDB code: $\left.1 d x 4\right)$ from the acetylcholinesterase type which display in addition to the two conserved disulphide, a third specific disulphide (arrow). In (c) is featured a representative from the paranitrobenzyl esterases group which do not feature any disulphide though two cysteines which are topologically equivalent to one highly conserved disulphide is present in the molecule.

Click here for file

[http://www.biomedcentral.com/content/supplementary/14726807-8-55-S3.tiff]

\section{Additional file 4}

Evolutionary dynamics of disulphides in the alpha/beta hydrolase serine carboxypeptidase-like family (SCOP code: $c$.69.1.5). Three superimposable members from this family are featured and which display one highly conserved disulphide (arrow). In (a) is featured a representative (SCOP domain: d1ac5_; PDB: 1ac5) from the serine carboxypeptidase II type and which displays two non-conserved disulphides. In (b) is featured a representative (SCOP domain: d1cpya_; PDB: $\underline{1 c p y}$ ) from the human carboxypeptidase $L$ type which display in addition to the conserved disulphide, four specific disulphides. In (c) is featured a representative from the human protective protein group which display in addition to the conserved disulphide, three specific disulphides.

Click here for file

[http://www.biomedcentral.com/content/supplementary/14726807-8-55-S4.tiff]

\section{Additional file 5}

Representation of Type-B carboxylesterase/lipase (SCOP domain: d1thga_; PDB: 1thg) from the fungal lipase family (SCOP family code: $\mathbf{c . 6 9 . 1 . 1 7 ) . ~ R e p r e s e n t a t i o n ~ o f ~ T y p e - B ~ c a r b o x y l e s t e r a s e / l i p a s e ~}$ (SCOP domain: d1 thga_PDB: 1thg) from the fungal lipase family (SCOP family code: $c$.69.1.17) that features two disulphides that are topologically equivalent to the two highly conserved disulphides from acetylcholinesterase-like family (SCOP family code: c.69.1.1).

Click here for file

[http://www.biomedcentral.com/content/supplementary/14726807-8-55-S5.tiff]

\section{Additional file 6}

Localization predictions by the three programs and the consensus starting from full-length sequences of proteins in the dataset. This table provides a comparison and consensus obtained by applying the three prediction servers for cellular localization.

Click here for file

[http://www.biomedcentral.com/content/supplementary/14726807-8-55-S6.pdf] 


\section{Additional file 7}

Literature survey of proteins highly predicted to be cytoplasmic by all three programs. This table provides the results of literature survey on comments of cellular localization for the protein chains, that are predicted to be cytoplasmic, by all three programs or by any two out of three programs. Click here for file

[http://www.biomedcentral.com/content/supplementary/14726807-8-55-S7.pdf]

\section{Additional file 8}

Conservation of disulphide bonds in protein chains highly predicted as cytoplasmic. This table provides the results of examination of conservation (or otherwise) of distinct disulphides for proteins that have been highly predicted to be cytoplasmic.

Click here for file

[http://www.biomedcentral.com/content/supplementary/14726807-8-55-S8.pdf]

\section{Acknowledgements}

RRT was supported by a PhD grant from the Conseil Régional de La Réunion. NS and RS thank the authorities of Universite de La Réunion for their visiting professorships. We thank Dr. Alexandre G. de Brevern for useful comments.

\section{References}

I. Richardson JS: The anatomy and taxonomy of protein structure. Adv Protein Chem 1981, 34:167-339.

2. Thornton JM: Disulphide bridges in globular proteins. J Mol Biol I98I, I 5 I (2):26I-287.

3. Ceroni A, Frasconi P, Passerini A, Vullo A: Predicting the Disulfide Bonding State of Cysteines with Combinations of Kernel Machines. The Journal of VLSI Signal Processing-Systems for Signal, Image, and Video Technology 2003, 35(3):287-295.

4. Chen YC, Lin YS, Lin CJ, Hwang JK: Prediction of the bonding states of cysteines using the support vector machines based on multiple feature vectors and cysteine state sequences. Proteins 2004, 55(4): 1036-1042.

5. Fariselli P, Riccobelli P, Casadio R: Role of evolutionary information in predicting the disulfide-bonding state of cysteine in proteins. Proteins 1999, 36(3):340-346.

6. Fiser A, Cserzo M, Tudos E, Simon I: Different sequence environments of cysteines and half cystines in proteins. Application to predict disulfide forming residues. FEBS Lett 1992, 302(2): $117-120$.

7. Fiser A, Simon I: Predicting the oxidation state of cysteines by multiple sequence alignment. Bioinformatics 2000, 16(3):25I-256.

8. Fiser A, Simon I: Predicting redox state of cysteines in proteins. Methods Enzymol 2002, 353:10-2I.

9. Frasconi P, Passerini A, Vullo A: A Two-Stage SVM Architecture for Predicting the Disulfide Bonding State of Cysteines. IEEE NNSP International Workshop: special session on signal processing and neural networks for bionformatics: 20022002.

10. Martelli PL, Fariselli P, Casadio R: Prediction of disulfide-bonded cysteines in proteomes with a hidden neural network. Proteomics 2004, 4(6): 1665-167I.

II. Martelli PL, Fariselli P, Malaguti L, Casadio R: Prediction of the disulfide bonding state of cysteines in proteins with hidden neural networks. Protein Eng 2002, I5(I 2):95I-953.

12. Martelli PL, Fariselli P, Malaguti L, Casadio R: Prediction of the disulfide-bonding state of cysteines in proteins at $88 \%$ accuracy. Protein Sci 2002, I I (I I):2735-2739.

13. Mucchielli-Giorgi MH, Hazout S, Tuffery P: Predicting the disulfide bonding state of cysteines using protein descriptors. Proteins 2002, 46(3):243-249.
14. Muskal SM, Holbrook SR, Kim SH: Prediction of the disulfidebonding state of cysteine in proteins. Protein Eng 1990, 3(8):667-672.

15. Passerini A, Frasconi P: Learning to discriminate between ligand-bound and disulfide-bound cysteines. Protein Eng Des Sel 2004, I 7(4):367-373.

16. Song JN, Wang ML, Li WJ, Xu WB: Prediction of the disulfidebonding state of cysteines in proteins based on dipeptide composition. Biochem Biophys Res Commun 2004, 3 I 8(I): 142-147.

17. Sharma D, Rajarathnam K: I3C NMR chemical shifts can predict disulfide bond formation. J Biomol NMR 2000, 18(2): 165-171.

18. Fariselli $P$, Casadio R: Prediction of disulfide connectivity in proteins. Bioinformatics 200I, I7(10):957-964.

19. Ferre F, Clote P: Disulfide connectivity prediction using secondary structure information and diresidue frequencies. Bioinformatics 2005, 21 (10):2336-2346.

20. Ferre F, Clote P: DiANNA: a web server for disulfide connectivity prediction. Nucleic Acids Res 2005:W230-232.

21. Tsai CH, Chen BJ, Chan CH, Liu HL, Kao CY: Improving disulfide connectivity prediction with sequential distance between oxidized cysteines. Bioinformatics 2005, 2 I (24):44I 6-44I9.

22. Vullo A, Frasconi P: A Recursive Connectionist Approach for Predicting Disulfide Connectivity in Proteins. Proceedings of the 18th ACM Symposium on Applied Computing: 2003 2003:66-7I.

23. Vullo $A$, Frasconi $P$ : Disulfide connectivity prediction using recursive neural networks and evolutionary information. Bioinformatics 2004, 20(5):653-659.

24. Zhao E, Liu HL, Tsai CH, Tsai HK, Chan CH, Kao CY: Cysteine separations profiles on protein sequences infer disulfide connectivity. Bioinformatics 2005, 2 I (8): | $4 \mid$ | -1420.

25. Hazes B, Dijkstra BW: Model building of disulfide bonds in proteins with known three-dimensional structure. Protein Eng 1988, 2(2): 119-125.

26. Sowdhamini R, Srinivasan N, Shoichet B, Santi D, Ramakrishnan C, Balaram P: Stereochemical modeling of disulfide bridges. Criteria for introduction into proteins by site-directed mutagenesis. Protein Eng 1989, 3(2):95-103.

27. Overington JP, Zhu ZY, Sali A, Johnson MS, Sowdhamini R, Louie GV, Blundell TL: Molecular recognition in protein families: a database of aligned three-dimensional structures of related proteins. Biochemical Society transactions I993, 2 I (Pt 3(3)):597-604.

28. Chuang CC, Chen CY, Yang JM, Lyu PC, Hwang JK: Relationship between protein structures and disulfide-bonding patterns. Proteins 2003, 53(1):I-5.

29. Mas JM, Aloy P, Marti-Renom MA, Oliva B, Blanco-Aparicio C, Molina MA, de Llorens R, Querol E, Aviles FX: Protein similarities beyond disulphide bridge topology. Journal of Molecular Biology 1998, 284(3):54|-548.

30. Abkevich VI, Shakhnovich El: What can disulfide bonds tell us about protein energetics, function and folding: simulations and bioninformatics analysis. J Mol Biol 2000, 300(4):975-985.

31. Berkmen M, Boyd D, Beckwith J: The nonconsecutive disulfide bond of Escherichia coli phytase (AppA) renders it dependent on the protein-disulfide isomerase, DsbC. J Biol Chem 2005, 280( I 2): I I 387-I I 394.

32. Betz SF: Disulfide bonds and the stability of globular proteins. Protein Sci 1993, 2(10): 155I-1558.

33. Clarke J, Hounslow AM, Bond CJ, Fersht AR, Daggett V: The effects of disulfide bonds on the denatured state of barnase. Protein Sci 2000, 9( I 2):2394-2404.

34. Matsumura M, Matthews BW: Control of enzyme activity by an engineered disulfide bond. Science 1989, 243(4892):792-794.

35. Matsumura M, Matthews BW: Stabilization of functional proteins by introduction of multiple disulfide bonds. Methods Enzymol 1991, 202:336-356.

36. Mayans O, Wuerges J, Canela S, Gautel M, Wilmanns M: Structural evidence for a possible role of reversible disulphide bridge formation in the elasticity of the muscle protein titin. Structure 200I, 9(4):33I-340.

37. Wedemeyer WJ, Welker E, Narayan M, Scheraga HA: Disulfide bonds and protein folding. Biochemistry 2000, 39(15):4207-4216.

38. Welker E, Wedemeyer W], Narayan M, Scheraga HA: Coupling of conformational folding and disulfide-bond reactions in oxidative folding of proteins. Biochemistry 200I, 40(3I):9059-9064.

39. Drakopoulou E, Vizzavona J, Neyton J, Aniort V, Bouet F, Virelizier H, Menez A, Vita C: Consequence of the removal of evolutionary 
conserved disulfide bridges on the structure and function of charybdotoxin and evidence that particular cysteine spacings govern specific disulfide bond formation. Biochemistry 1998, 37(5):|292-|30|.

40. Altschuh D, Vernet T, Berti P, Moras D, Nagai K: Coordinated amino acid changes in homologous protein families. Protein Eng 1988, 2(3): 193-199.

41. Kreisberg R, Buchner V, Arad D: Paired natural cysteine mutation mapping: Aid to constraining models of protein tertiary structure. Protein Sci 1995, 4(I I):2405-24I0.

42. Murzin AG, Brenner SE, Hubbard T, Chothia C: SCOP: a structural classification of proteins database for the investigation of sequences and structures. J Mol Biol 1995, 247(4):536-540.

43. Kidera A, Inaka K, Matsushima M, Go N: Response of dynamic structure to removal of a disulfide bond: normal mode refinement of C77A/C95A mutant of human lysozyme. Protein Sci 1994, 3(1):92-102.

44. Hinck AP, Truckses DM, Markley JL: Engineered disulfide bonds in staphylococcal nuclease: effects on the stability and conformation of the folded protein. Biochemistry 1996 35(32): $10328-10338$

45. Creighton TE: Disulphide bonds and protein stability. Bioessays 1988, 8(2):57-63.

46. Dill KA: Theory for the folding and stability of globular proteins. Biochemistry 1985, 24(6): I50 I-1509.

47. Wetlaufer DB: Folding of protein fragments. Adv Protein Chem I 98I, 34:6I-92.

48. Richardson JS, Richardson DC: The de novo design of protein structures. Trends Biochem Sci 1989, I4(7):304-309.

49. Harrison PM, Sternberg MJE: The Disulphide [beta]-Cross: From Cystine Geometry and Clustering to Classification of Small Disulphide-rich Protein Folds. Journal of Molecular Biology 1996, 264(3):603-623.

50. Jing $\mathrm{H}, \mathrm{Xu}$ Y, Carson M, Moore D, Macon KJ, Volanakis JE, Narayana SV: New structural motifs on the chymotrypsin fold and their potential roles in complement factor B. Embo J 2000 19(2): 164-173.

5I. Wang X, Terzyan S, Tang J, Loy JA, Lin X, Zhang XC: Human plasminogen catalytic domain undergoes an unusual conformational change upon activation. J Mol Biol 2000, 295(4):903-9l4.

52. Parry MA, Jacob U, Huber R, Wisner A, Bon C, Bode W: The crystal structure of the novel snake venom plasminogen activator TSV-PA: a prototype structure for snake venom serine proteinases. Structure 1998, 6(9): I 195-1 206.

53. Lougheed JC, Holton JM, Alber T, Bazan JF, Handel TM: Structure of melanoma inhibitory activity protein, a member of a recently identified family of secreted proteins. Proc Natl Acad Sci USA 200I, 98( I 0):55I5-5520.

54. Derman Al, Beckwith J: Escherichia coli alkaline phosphatase fails to acquire disulfide bonds when retained in the cytoplasm. Journal of bacteriology 199I, I73(23):77|9-7722

55. Kadokura H, Katzen F, Beckwith J: Protein disulfide bond formation in prokaryotes. Annual review of biochemistry 2003, 72: III-135

56. Prinz WA, Aslund F, Holmgren A, Beckwith J: The Role of the Thioredoxin and Glutaredoxin Pathways in Reducing Protein Disulfide Bonds in the Escherichia coli Cytoplasm. J Biol Chem 1997, 272(25): I566I-I5667.

57. Choi H-J, Kim S-J, Mukhopadhyay P, Cho S, Woo J-R, Storz G, Ryu SE: Structural Basis of the Redox Switch in the OxyR Transcription Factor. Cell 200I, I05(I): I03-II3

58. Jakob U, Muse W, Eser M, Bardwell JCA: Chaperone Activity with a Redox Switch. Cell 1999, 96(3):34I-352.

59. Toth EA, Worby C, Dixon JE, Goedken ER, Marqusee S, Yeates TO: The crystal structure of adenylosuccinate lyase from Pyrobaculum aerophilum reveals an intracellular protein with three disulfide bonds. Journal of Molecular Biology 2000 $30 I(2): 433-450$

60. Littler DR, Harrop SJ, Fairlie WD, Brown LJ, Pankhurst G], Pankhurst S, DeMaere MZ, Campbell TJ, Bauskin AR, Tonini R, et al:: The intracellular chloride ion channel protein CLICI undergoes a redox-controlled structural transition. J Biol Chem 2004, 279(10):9298-9305.

61. Knaus KJ, Morillas M, Swietnicki W, Malone M, Surewicz WK, Yee VC: Crystal structure of the human prion protein reveals a mechanism for oligomerization. Nat Struct Biol 200I, 8(9):770-774.

62. Bar J, Weber A, Hoffmann T, Stork J, Wermann M, Wagner L, Aust S, Gerhartz B, Demuth HU: Characterisation of human dipeptidyl peptidase IV expressed in Pichia pastoris. A structural and mechanistic comparison between the recombinant human and the purified porcine enzyme. Biol Chem 2003, 384( I 2): I $553-1563$.

63. Krauspenhaar R, Eschenburg S, Perbandt M, Kornilov V, Konareva N Mikailova I, Stoeva S, Wacker R, Maier T, Singh T, et al.: Crystal structure of mistletoe lectin I from Viscum album. Biochem Biophys Res Commun 1999, 257(2):4I8-424.

64. Gruber K, Klintschar G, Hayn M, Schlacher A, Steiner W, Kratky C: Thermophilic xylanase from Thermomyces lanuginosus: high-resolution X-ray structure and modeling studies. Biochemistry 1998, 37(39): I 3475-13485

65. Yamamoto A, Tomoo K, Miyagawa $\mathrm{H}$, Takaoka $\mathrm{Y}$, Sumiya S, Kitamura $\mathrm{K}$, Ishida T: Molecular dynamics simulations of bovine cathepsin B and its complex with CA074. Chem Pharm Bull (Tokyo) 2000, 48(4):480-485.

66. Anderson DH, Harth G, Horwitz MA, Eisenberg D: An interfacial mechanism and a class of inhibitors inferred from two crystal structures of the Mycobacterium tuberculosis $30 \mathrm{kDa}$ major secretory protein (Antigen 85B), a mycolyl transferase. J Mol Biol 200I, 307(2):67I-68I.

67. Xia Z, Dai W, Zhang Y, White SA, Boyd GD, Mathews FS: Determination of the gene sequence and the three-dimensional structure at 2.4 angstroms resolution of methanol dehydrogenase from Methylophilus W3AI. J Mol Biol 1996, 259(3):480-50I.

68. Smith CA, Toogood HS, Baker HM, Daniel RM, Baker EN: Calciummediated thermostability in the subtilisin superfamily: the crystal structure of Bacillus Ak.I protease at I.8 A resolution. I Mol Biol 1999, 294(4): I027-1040.

69. Gong W, Zhu X, Liu S, Teng M, Niu L: Crystal structures of acutolysin A, a three-disulfide hemorrhagic zinc metalloproteinase from the snake venom of Agkistrodon acutus. J Mol Biol 1998, 283(3):657-668

70. van Vlijmen HWT, Gupta A, Narasimhan LS, Singh J: A Novel Database of Disulfide Patterns and its Application to the Discovery of Distantly Related Homologs. Journal of Molecular Biology 2004, 335(4): 1083-1092.

7I. Xiao T, DeCamp DL, Spran SR: Structure of a rat alpha I-macroglobulin receptor-binding domain dimer. Protein Sci 2000 , 9(10): 1889-1897.

72. Serre L, Pereira de Jesus K, Zelwer C, Bureaud N, Schoentgen F, Benedetti $\mathrm{H}$ : Crystal structures of YBHB and YBCL from Escherichia coli, two bacterial homologues to a Raf kinase inhibitor protein. J Mol Biol 200I, 3 I0(3):617-634.

73. Colbert CL, Couture MM, Eltis LD, Bolin JT: A cluster exposed: structure of the Rieske ferredoxin from biphenyl dioxygenase and the redox properties of Rieske Fe-S proteins. Structure 2000, 8(I 2): 1267-I278.

74. Gugolya Z, Dosztanyi Z, Simon I: Interresidue interactions in protein classes. Proteins 1997, 27(3):360-366.

75. Dosztanyi Z, Fiser A, Simon I: Stabilization centers in proteins: identification, characterization and predictions. J Mol Biol 1997, 272(4):597-6/2.

76. Levitt $M$, Chothia C: Structural patterns in globular proteins. Nature 1976, 26 I (556 I):552-558.

77. Thangudu RR, Vinayagam A, Pugalenthi G, Manonmani A, Offmann B, Sowdhamini R: Native and modeled disulfide bonds in proteins: knowledge-based approaches toward structure prediction of disulfide-rich polypeptides. Proteins 2005, 58(4):866-879.

78. Dani VS, Ramakrishnan C, Varadarajan R: MODIP revisited: reevaluation and refinement of an automated procedure for modeling of disulfide bonds in proteins. Protein Eng 2003, 16(3): 187-193

79. Panchenko AR, Wolf YI, Panchenko LA, Madej T: Evolutionary plasticity of protein families: coupling between sequence and structure variation. Proteins 2005, 61 (3):535-544.

80. Wilson CA, Kreychman J, Gerstein M: Assessing annotation transfer for genomics: quantifying the relations between protein sequence, structure and function through traditional and probabilistic scores. I Mol Biol 2000, 297(I):233-249. 
8I. Torrance JW, Bartlett G], Porter CT, Thornton JM: Using a library of structural templates to recognise catalytic sites and explore their evolution in homologous families. J Mol Biol 2005, 347(3):565-58I.

82. Dai Y, Tang JG: Intra-A chain disulfide bond (A6-II) of insulin is essential for displaying its activity. Biochem Mol Biol Int 1994, 33(6): 1049-1053.

83. Eigenbrot C, Randal M, Kossiakoff AA: Structural effects induced by removal of a disulfide-bridge: the $X$-ray structure of the C30A/C5 I A mutant of basic pancreatic trypsin inhibitor at I.6 A. Protein Eng 1990, 3(7):591-598.

84. Li T, Yamane H, Arakawa T, Narhi LO, Philo J: Effect of the intermolecular disulfide bond on the conformation and stability of glial cell line-derived neurotrophic factor. Protein Eng 2002, I 5(I):59-64

85. Doolittle RF: The genealogy of some recently evolved vertebrate proteins. Trends in Biochemical Sciences 1985, 10(6):233-237.

86. Mallick P, Boutz DR, Eisenberg D, Yeates TO: Genomic evidence that the intracellular proteins of archaeal microbes contain disulfide bonds. Proc Natl Acad Sci USA 2002, 99(15):9679-9684.

87. Ladenstein R, Ren $B$ : Reconsideration of an early dogma, saying "there is no evidence for disulfide bonds in proteins from archaea". Extremophiles 2008, I 2(I):29-38.

88. Nakashima H, Nishikawa K: Discrimination of intracellular and extracellular proteins using amino acid composition and residue-pair frequencies. J Mol Biol 1994, 238(I):54-6I.

89. Nishikawa K, Kubota $Y$, Ooi T: Classification of proteins into groups based on amino acid composition and other characters. II. Grouping into four types. Journal of biochemistry 1983, 94(3):997-1007.

90. Thangudu RR, Sharma P, Srinivasan N, Offmann B: Analycys: a database for conservation and conformation of disulphide bonds in homologous protein domains. Proteins 2007, 67(2):255-26I.

91. Berman HM, Westbrook J, Feng Z, Gilliland G, Bhat TN, Weissig H, Shindyalov IN, Bourne PE: The Protein Data Bank. Nucleic Acids Res 2000, 28(I):235-242.

92. Emanuelsson O, Nielsen H, Brunak S, von Heijne G: Predicting subcellular localization of proteins based on their $\mathbf{N}$-terminal amino acid sequence. J Mol Biol 2000, 300(4): 1005-1016.

93. Nakai K, Horton P: PSORT: a program for detecting sorting signals in proteins and predicting their subcellular localization. Trends Biochem Sci 1999, 24(I):34-36.

94. Hua S, Sun Z: Support vector machine approach for protein subcellular localization prediction. Bioinformatics 200I, | 7(8):72|-728.

95. Russell RB, Barton GJ: Multiple protein sequence alignment from tertiary structure comparison: assignment of global and residue confidence levels. Proteins 1992, 14(2):309-323.

96. Argos P, Rossmann MG: A method to determine heavy-atom positions for virus structures. Acta Crystallographica 1976, B32:2975-2979.

97. Kabsch W, Sander C: Dictionary of protein secondary structure: pattern recognition of hydrogen-bonded and geometrical features. Biopolymers 1983, 22(12):2577-2637.

98. Lee B, Richards FM: The interpretation of protein structures: estimation of static accessibility. I Mol Biol I 97I , 55(3):379-400.

99. Hubbard SJ, Thornton JM: Naccess V2.I.I, Atomic solvent accessible area calculations. In Department of biochemistry and molecular biology University college London; 1993.

100. Pintar A, Carugo O, Pongor S: DPX: for the analysis of the protein core. Bioinformatics 2003, 19(2):313-314.
Publish with Biomed Central and every scientist can read your work free of charge

"BioMed Central will be the most significant development for disseminating the results of biomedical research in our lifetime. "

Sir Paul Nurse, Cancer Research UK

Your research papers will be:

- available free of charge to the entire biomedical community

- peer reviewed and published immediately upon acceptance

- cited in PubMed and archived on PubMed Central

- yours - you keep the copyright
BioMedcentral 